\title{
YZiCS: Unveiling the Quenching History of Cluster Galaxies Using Phase-space Analysis
}

\author{
Jinsu Rhee ${ }^{1}(1)$, Rory Smith ${ }^{2}$, Hoseung Choi $^{3}{ }^{1}$, Emanuele Contini ${ }^{4}$, S. Lyla Jung ${ }^{1,5}$, San Han $^{1}$, and Sukyoung K. $\mathrm{Yi}^{1}{ }^{1}$ \\ ${ }^{1}$ Department of Astronomy and Yonsei University Observatory, Yonsei University, Seoul 03722, Republic of Korea; jinsu.rhee@yonsei.ac.kr \\ ${ }^{2}$ Korea Astronomy and Space Science Institute, 776, Daedeokdae-ro, Yuseong-gu, Daejeon, 34055, Republic of Korea \\ ${ }^{3}$ Institute of Theoretical Astrophysics, University of Oslo, Postboks 1029, Blindern, NO-0315 Oslo, Norway \\ ${ }^{4}$ School of Astronomy and Space Science, Nanjing University, Nanjing 210093, People's Republic of China \\ ${ }^{5}$ Research School of Astronomy \& Astrophysics, Australian National University, Canberra, ACT 2611, Australia \\ Received 2019 September 26; revised 2020 January 22; accepted 2020 February 5; published 2020 March 16
}

\begin{abstract}
We used the time since infall (TSI) of galaxies, obtained from the Yonsei Zoom-in Cluster Simulation, and the star formation rate (SFR) from the Sloan Digital Sky Survey Data Release 10 to study how quickly the star formation of disk galaxies is quenched in cluster environments. We first confirm that both simulated and observed galaxies are consistently distributed in phase space. We then hypothesize that the TSI and SFR are causally connected; thus, both the TSI and SFR of galaxies at each position of phase space can be associated through abundance matching. Using a flexible model, we derive the star formation history (SFH) of cluster galaxies that best reproduces the relationship between the TSI and SFR at $z \sim 0.08$. According to this SFH, we find that galaxies with $M_{*}>10^{9.5} M_{\odot}$ generally follow the so-called "delayed-then-rapid" quenching pattern. Our main results are as follows: (i) part of the quenching takes place outside clusters through mass quenching and preprocessing. The e-folding timescale of this "ex situ quenching phase" is roughly 3 Gyr with a strong inverse mass dependence. (ii) The pace of quenching is maintained roughly for 2 Gyr ("delay time") during the first crossing time into the cluster. During the delay time, quenching remains gentle, probably because gas loss happens primarily on hot and neutral gases. (iii) Quenching becomes more dramatic (e-folding timescale of roughly 1 Gyr) after delay time, probably because ram pressure stripping is strongest near the cluster center. Counterintuitively, more massive galaxies show shorter quenching timescales mainly because they enter their clusters with lower gas fractions due to ex situ quenching.
\end{abstract}

Unified Astronomy Thesaurus concepts: Galaxy evolution (594)

\section{Introduction}

In this new era of large-scale observational surveys and cosmological simulations with hydrodynamic calculations, a standard view of the baryon cycle for cluster galaxies has been, at least qualitatively, established. For an infalling galaxy, for example, the supply of external gas begins to diminish even beyond the outskirts of clusters (e.g., Behroozi et al. 2014; McGee et al. 2014) as the loosely bound galactic hot gas, which could be potential fuel for star formation, is easily stripped away, starving the galaxy ("starvation/strangulation"; Larson et al. 1980; Balogh et al. 2000). In the case of more massive galaxies, their larger halo mass $\left(M_{\text {halo }}>10^{12} M_{\odot}\right)$ induces virial shock heating for the infalling gas, which prohibits the infalling gas from forming stars ("halo quenching"; Binney 1977; Birnboim \& Dekel 2003; Kereš et al. 2005; Woo et al. 2013). After crossing the cluster boundary, the hydrodynamical interaction with the surrounding intracluster medium (ICM; "ram pressure") is a key process that influences a galaxy's interstellar medium (ISM; Gunn \& Gott 1972; Chung et al. 2007; Bekki 2014; Steinhauser et al. 2016), and gravitational tides from the cluster's deep potential well ("tidal stripping") cause the ISM, dark matter (DM), and stars of the galaxy to be stripped away (e.g., Gao et al. 2004; Limousin et al. 2009; Smith et al. 2016). Furthermore, continuous tidal encounters with nearby cluster galaxies ("harassment"; Moore et al. 1996, 1998; Smith et al. 2010, 2013, 2015) and galaxy mergers (Toomre \& Toomre 1972) may leave distorted features in the components of a galaxy (see also Sheen et al. 2012; Yi et al. 2013). Previous group-mass hosts $\left(\sim M_{\text {halo }}^{12-13}\right)$ can be a major influence on a galaxy prior to infall into the cluster ("preprocessing"; Mihos 2004; De Lucia et al. 2012; Han et al. 2018; Jung et al. 2018). Moreover, internal feedback processes driven by the active galactic nucleus (AGN), stellar winds, and/or supernovae can trigger outflows of a galaxy's gas reservoir (Larson 1974; Croton et al. 2006; McGee et al. 2014), a mechanism that is often referred to as "mass quenching" (Peng et al. 2010).

Observationally speaking, it is well known that dense regions are preferred by early-type galaxies, a result known as the morphology-density relation (Dressler 1980). Moreover, numerous galaxies deficient in atomic and/or molecular gas are detected in the clusters of galaxies (Gavazzi 1987; Fumagalli et al. 2009; Boselli et al. 2014b). Because of ram pressure, certain cluster galaxies present truncated or stripped features in their gas components (Koopmann \& Kenney 2004; Chung et al. 2007; Lee \& Chung 2018), which highlights the outside-in quenching process they undergo (Koopmann \& Kenney 2004; Cortese et al. 2012; Fossati et al. 2018; Jaffé et al. 2018). For large statistical samples of cluster galaxies, it has been found that the colors of their stellar populations are associated with the local density of galaxies such that they become older and redder with increasing density (e.g., Hogg et al. 2004; Lemaux et al. 2019). More directly, the star formation rate (SFR) by itself decreases with increasing local galaxy density and/or with decreasing clustocentric distance (Balogh et al. 2000; Lewis et al. 2002; Gómez et al. 2003; Kauffmann et al. 2004). However, despite the impressive groundwork of statistical studies, the primary origin of passive galaxies in clusters remains an unresolved issue because of the complex nonlinearity of the various quenching processes.

Over the past few decades, various quenching models for cluster galaxies have been proposed to simplify the nonlinearity. 
The "rapid quenching model" was one of the first models in which cluster galaxies were quenched within a very short timescale ( $\lesssim 1 \mathrm{Gyr})$, immediately after being accreted into clusters (e.g., Balogh et al. 2004; Muzzin et al. 2012). The literature primarily focused on the fact that the intensity of star formation (SF) weakly depends on the environment for starforming galaxies, although the fraction of star-forming objects show a strong environmental dependence. This alone implies that the transformation into passive galaxies occurs on short timescales (Peng et al. 2010; McGee et al. 2011; Mok et al. 2013, 2014; Muzzin et al. 2014; see also De Lucia et al. 2012 and Tyler et al. 2013 for counterarguments).

Based on more recent observations, however, the mean SFR of star-forming galaxies is observed to be more suppressed in dense environments, compared to their field counterparts at a fixed stellar mass. This indicates that the quenching process is slow enough to be detected (Wolf et al. 2009; Vulcani et al. 2010; McGee et al. 2011; Haines et al. 2013; Paccagnella et al. 2016; Rodríguez-Muñoz et al. 2019). Combining a semianalytic approach with data from large-scale surveys, strangulation/starvation is considered to be a possible physical mechanism behind the "slow quenching scenario" (e.g., Weinmann et al. 2009, 2010; von der Linden et al. 2010; De Lucia et al. 2012; Taranu et al. 2014).

The concept introduced to reconcile the two ideas is delay time, which was suggested by Wetzel et al. (2013), in which galaxies remain unaffected by the cluster environment for a few gigayears after becoming a satellite (see also McCarthy et al. 2008; Haines et al. 2015; Paccagnella et al. 2016). After the delay time, galaxies are quickly quenched, that is, galaxies are quenched in the manner of "delayed then rapid." This quenching model has been consistently supported by many studies due to its suitability for describing the quenching of galaxies (Wetzel et al. 2013; McGee et al. 2014; Mok et al. 2014; Tal et al. 2014; Balogh et al. 2016; Fossati et al. 2017; Foltz et al. 2018). Yet, there are still certain results that are inconsistent with the delayed-then-rapid model. For example, by noting the gradual decrease of the SFR of the star-forming galaxies inside clusters, Haines et al. (2015) proposed the "slow-then-rapid," model adopting a slightly faster quenching during the "delay phase" (see also Maier et al. 2019).

Quenching models are constrained by comparing the observations with theoretical predictions. In multiple studies, the preferred observational parameters include the fraction of passive or starforming galaxies (e.g., Mok et al. 2014; Oman \& Hudson 2016; Fossati et al. 2017), the environmental/mass-quenching efficiencies (e.g., Peng et al. 2010; Balogh et al. 2016; Darvish et al. 2016; Lemaux et al. 2019), the main-sequence relation between the SFR and stellar mass (e.g., Peng et al. 2010; Paccagnella et al. 2016), the color/SFR distribution (e.g., Haines et al. 2013; Wetzel et al. 2013; Foltz et al. 2018), and other galactic properties (e.g., Weinmann et al. 2009; Taranu et al. 2014).

The purpose of our investigation is to explore the dominant quenching processes by focusing on the quenching timescales of cluster galaxies. Following the tradition in the field, we compare the properties of observed galaxies with theoretical predictions from numerical simulations. We introduce a new approach of using abundance matching between observed data (SFR) and theoretical prediction through a phase-space analysis.

A phase-space diagram is a plot of the velocity versus distance of cluster galaxies, both of which are measured with respect to the cluster's center. According to previous simulation studies (e.g., Gill et al. 2005; Oman et al. 2013; Rhee et al. 2017), cluster galaxies, during their infall into a cluster, tend to follow a common path through this phase space (see Figure 1 in Rhee et al. 2017 for a summary). This premise is supported by the fact that infalling galaxies share favored orbital parameters (Wetzel 2011) and the crossing times of clusters are similar regardless of cluster mass and stellar mass (Jung et al. 2018; Lotz et al. 2019).

Following common trajectories in phase space likely leaves common traces on galaxy properties in each position of the phase space and also along the orbital path. Numerous investigations have indeed considered the distribution of galaxies on a phasespace plane. To infer the physical processes occurring during the infall, the distribution often associated with galaxies' properties is used to connect the properties with galaxies' orbital states. Examples include quenching of galaxies (Mahajan et al. 2011; Oman \& Hudson 2016), gas stripping because of ram pressure (Hernández-Fernández et al. 2014; Jaffé et al. 2015, 2018; Yoon et al. 2017), and the assembly history of infalling satellites/groups (Oman et al. 2013; Einasto et al. 2018a, 2018b; Lisker et al. 2018; Adhikari et al. 2019). In particular, Pasquali et al. (2019) calculated the mean values of the time since infall (TSI) of the populations of observed galaxies in different zones in a projected phase space and then studied how their observed mean properties (such as sSFR, age, and metallicity) correlate with their mean TSI (see also Smith et al. 2019).

We instead divide a projected phase-space diagram into a grid of pixels. Then, rather than using the mean value within each pixel, we build density functions of both TSI (derived from simulations) and SFR (from observations) and associate them through abundance matching to derive the SFR-TSI relationship in each pixel (see also Hearin \& Watson 2013; Behroozi et al. 2019). By combining the SFR-TSI relations from all pixels, we achieve the overall relationship between SFR and TSI, which in turn allows us to quantify the quenching timescales for cluster galaxies.

This paper is organized as follows. In Section 2, we describe the numerical simulation data used to obtain the TSI information for cluster galaxies (Section 2.1) and the observed cluster catalog and physical properties of satellite galaxies (Section 2.2). In Section 3, we define the projected phase-space coordinates for satellites and illustrate their distributions in projected phase space (Section 3.1) and then show how the probability density functions of TSI (Section 3.2) and SFR (Section 3.3) are derived. In Section 4, we demonstrate how we obtain the SFR-TSI relationship from the derived density functions (Section 4.1), and then we use this to measure the parameters of our quenching model (Section 4.2). We then discuss the resultant quenching parameters in Section 5. In Section 6, we summarize what our results say about the dominant quenching process for satellite galaxies (Section 6.1) and their quenching times inside clusters (Section 6.2), and provide further discussions.

\section{Sample}

\subsection{Numerical Simulation Data}

\subsubsection{Cosmological Hydrodynamic Simulation}

We used the simulation data of clusters and their galaxies from the Yonsei Zoom-in Cluster Simulation (YZiCS), a cosmological hydrodynamic zoom-in simulation on galaxy clusters using the adaptive mesh refinement code, RAMSES 
(Teyssier 2002). A more detailed description is given in Choi \& Yi (2017) and only a brief summary of the simulation is provided here.

We first ran a large, DM-only, cosmological simulation that has a cubic shape with a side length of $200 \mathrm{Mpc} \mathrm{h}^{-1}$ and adopted the WMAP7 cosmology (Komatsu et al. 2011): $\Omega_{\mathrm{M}}=0.272, \Omega_{\Lambda}=$ $0.728, H_{0}=70.4 \mathrm{~km} \mathrm{~s}^{-1} \mathrm{Mpc}^{-1}, \sigma_{8}=0.809$, and $n=0.963$. We assumed these cosmological parameters throughout the study. We then selected 15 high-density regions with a virial mass above $5 \times 10^{13} M_{\odot}$ at $z=0$ and performed zoom-in simulations, including gas and hydrodynamic recipes this time. The size of the zoom-in region encompasses all the particles within three virial radii of a cluster at $z=0$.

We adopted the baryon prescriptions of Dubois et al. (2012), including gas cooling and heating, SF, and stellar and AGN feedback models (see also Dubois et al. 2014). These models were originally applied in the Horizon-AGN simulation (Dubois et al. 2014), which has been demonstrated to faithfully reproduce the basic observable features of galaxies across a broad redshift range, including the cosmic evolution of the stellar luminosity function, star formation main sequence (MS), galaxy color distributions, cosmic star formation history (SFH), the morphological diversity, and the size-mass relation (Dubois et al. 2016; Kaviraj et al. 2017).

The maximum resolution of the simulation is roughly $760 \mathrm{pc} \mathrm{h}^{-1}$ for force calculations, $8 \times 10^{7} M_{\odot}$ for DM particle mass, and $5 \times 10^{5} M_{\odot}$ for stellar particle mass. We use the data up to $z=3$ in which the minimum time gap between two adjacent snapshots is roughly $77 \mathrm{Myr}$.

\subsubsection{Clusters and Galaxies in YZiCS}

We use 15 clusters and their nearby galaxies (within three virial radii at $z=0$ ) as our numerical sample. We will refer to them as "YZiCS clusters" and "YZiCS galaxies," respectively. All clusters range in virial mass from $5 \times 10^{13}$ to $1 \times 10^{15} M_{\odot}$, and more detailed information about the individual clusters is given in Table 1 of Rhee et al. (2017). To identify DM halos and galaxies in each snapshot, we used the HALOMAKER and the GALAXYMAKER codes, respectively, based on the AdaptaHOP method (Aubert et al. 2004; Tweed et al. 2009).

To be specific, the HALOMAKER code is based on the smoothed density field derived at each DM particle. The volumes within which the mean density is greater than a certain level of background density are treated as halo candidates. Then, from this volume, the largest ellipsoid within which the virial theorem is satisfied is considered as the halo. Subsequently, the virial mass and the virial radius are defined as the total mass within the ellipsoid and the radius of the ellipsoid, respectively, and the center of the halo is defined as the location of the peak density. The largest and most massive halos in each zoom region are defined as the cluster halos of our samples.

Galaxies are identified in a similar manner in the GALAXYMAKER code but using stellar particles. We used a cut of 200 stellar particles to define a galaxy corresponding to $M_{*} \sim$ $10^{8} M_{\odot}$. However, to minimize numerical noise, we used the galaxies with $M_{*}>10^{9.5} M_{\odot}($ at $z=0)$. Consequently, there are a total of 2278 galaxies at $z=0$ in the main sample.

Then, for fair comparison with the observed galaxy sample, we considered the flux limitation of the Sloan Digital Sky Survey (SDSS) galaxies $\left(m_{r}<17.77\right)$ and the projection effects on the SDSS galaxies. We used the YZiCS galaxies in snapshots with different redshifts to limit the galaxy sample based on their $r$-band magnitude at the corresponding redshift, and we selected multiple lines of sight to mimic the projection effects on the simulated galaxies in each snapshot (Section 3.1.1). Furthermore, the YZiCS galaxies within a given area of the projected phase space are selected to reduce contamination from interlopers (Section 3.1.3). Figure 1(b) shows the stellar mass function of the YZiCS galaxies after considering the multiple projections and luminosity cuts (red dashed line).

Compared to the SDSS galaxies, the YZiCS galaxies seem lower in mass roughly by a factor of 2.2 , perhaps due to an over-quenching issue. Hence, when we performed abundance matching, we artificially multiplied the stellar mass of YZiCS galaxies by the same factor. The modified stellar mass function of YZiCS galaxies is shown as the red solid line in Figure 1(b). This modification factor generates good agreement between the two stellar mass functions for galaxies above $2 \times 10^{10} M_{\odot}$ while there are more YZiCS galaxies in the lower mass range, most possibly because of the low completeness of faint galaxies in observations. Regarding the correction factor, we admit that the choice of the value is somewhat uncertain, yet it does not affect the conclusion much. For example, if we do not apply the mass modification factor of 2.2, the quenching timescales we will derive later as the main results are altered by $20 \%$.

\subsection{Observational Data}

\subsubsection{Clusters and Galaxies in SDSS}

For the observed cluster and galaxy samples, we used the cluster and the galaxy catalogs provided by Tempel et al. (2014). To determine the clusters, they used flux-limited galaxies in SDSS DR 10 (Ahn et al. 2014) from $z=0$ to $z=0.2$, using the Friends-of-Friends method. Initially, the cluster catalog contained 82,458 groups/clusters, and we used four properties (richness, R.A., decl., and redshift) for our analysis.

Among 82,458 groups or clusters, we avoided unreliable groups/clusters by removing samples with the value of richness or the number of galaxies inside the projected phase space less than 20 (see Section 3.1.2 for the detailed definition of projected phase space). The numerical limit is arbitrarily selected; however, we confirmed that the primary results of this analysis are invariant to different numerical values for the limit. After this stage, 793 groups/clusters remained. We then confined SDSS groups/clusters to the same lower mass limit as in $\mathrm{YZiCS}\left(>5 \times 10^{13} M_{\odot}\right)$. With this cut, 415 groups/ clusters remained with a median redshift of 0.08 and the highest redshift of 0.166 , and their virial mass distribution is shown as the gray histogram in Figure 1(a). Hereafter, we will refer to this sample as the "SDSS clusters." In the following section, we will explain how the virial mass of the SDSS clusters is defined. Using the $r$-band luminosity-weighted mean values of the R.A. and decl. of the member galaxies, we defined the central position of each cluster; in this step, we used the membership identification conducted by Tempel et al. (2014).

Similarly, we used SDSS DR 10 galaxies up to $z=0.2$ in the galaxy catalog of Tempel et al. (2014), in which there were originally 588,193 galaxies. The catalog provides a series of galaxy properties, and then we utilized R.A., decl., redshift, morphology, and $k+e$-corrected absolute magnitudes of the $r$-band filter from the catalog. The morphological classification was performed by Huertas-Company et al. (2011), in which they conducted machine learning with three galactic properties 

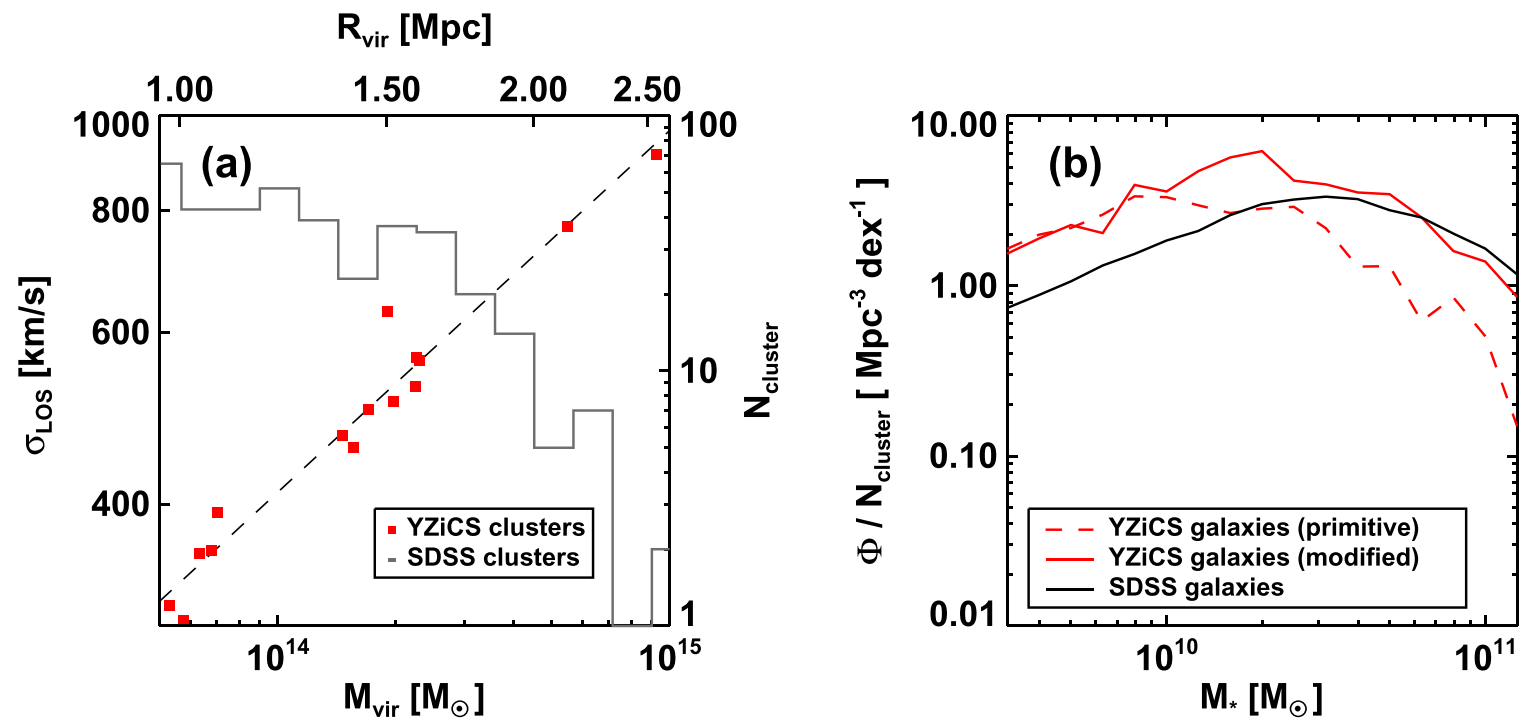

Figure 1. (a) The relationship between virial mass and line-of-sight velocity dispersion for the YZiCS cluster sample (red squares). The black dashed line indicates the linear fitting of the relationship. The gray histogram shows the virial mass distribution of clusters from the SDSS, and their masses are computed using the fitting line from their velocity dispersion (see the text for details). On the top axis, the virial radius corresponding to each virial mass is noted. In this manner, we have a cluster mass and virial radius similar to the observations for a given velocity dispersion. (b) Stellar mass functions of the galaxy samples within a given location in the projected phase space (see Section 3.1.3). Each stellar mass function is normalized by the comoving volume of each cluster (within 1.5 virial radii) and the total number of clusters. The red dashed line is the stellar mass function of the primitive YZiCS galaxies, and the red solid line denotes the YZiCS galaxies with mass modified by a factor of 2.2 (see text for details). The black solid line is the stellar mass function of the SDSS galaxies. By modifying stellar masses, the YZiCS galaxies' stellar mass function resembles that of the SDSS above $2 \times 10^{10} M_{\odot}$. The low completeness of the faint SDSS galaxies might be a source of the discrepancy of the two stellar mass functions in the lower mass range.

(color, axis ratio, and concentration index) and determined a probability of the $\sim 700,000$ SDSS galaxies belonging to each morphological class (E, S0, Sab, Scd). We consider the class with the highest probability as the morphology of individual galaxies. We adopted the stellar mass and SFR of SDSS galaxies from Salim et al. (2016), who used a spectral energy distribution (SED) fitting to the UV and optical photometries combined with the mid-IR and $\mathrm{H} \alpha$ emissions, assuming a Chabrier initial mass function (Chabrier 2003). For the comparison with YZiCS, we used only the SDSS galaxies with spectroscopic data and a mass limit of $>10^{9.5} M_{\odot}$.

In addition, because our study is primarily focused on the star-forming quenching history, we used only disk galaxies (Sab, Scd, and S0) for our analysis. Because the morphology classification is based on both galactic colors and actual shapes of galaxies, the selected sample will include blue spiral to quenched disk galaxies. We made this sample selection because disk galaxies might be expected to show remarkable signs of quenching driven by the cluster environment, i.e., elliptical galaxies are not a good sample for our purposes due to their early quenching in SF. To avoid introducing a progenitor bias for disk galaxies, we had to assume that the morphological transition from disk to elliptical galaxies will be a rare event in the clusters. Indeed, $<10 \%$ of cluster members are found to undergo galaxy-galaxy mergers (e.g., Lee et al. 2018), and the timescale for structural transformation is expected to be longer than the dynamical timescales inside clusters (e.g., Kelkar et al. 2019).

The final sample included 17,879 disk galaxies within host clusters (see Section 3.1.2 for their membership identification). The stellar mass function of all member galaxies (within a specified area of the projected phase space; see Section 3.1.3) is shown in Figure 1(b) using the black solid line.

\subsubsection{Normalizing Factors of the Phase-space Diagram}

Phase-space coordinates are typically normalized by dynamical mass indicators (virial radius and velocity dispersion in this and many other studies) such that clusters of widely varying masses can be stacked within a single diagram. The coordinates, therefore, sensitively depend on how one defines these normalization factors: e.g., the virial radius in Girardi et al. (1998) can significantly differ from that given in Navarro et al. (1996). Therefore, one should carefully choose normalization factors when comparing observed data and theoretical models in the same diagram.

To fine-tune the normalization factors of the YZiCS and SDSS clusters, we start from a directly observable property: the line-ofsight velocity dispersion of the SDSS clusters, $\sigma_{\mathrm{LOS}}$. Initially, we used the virial radius and velocity dispersion given in the cluster catalog and identified all the members of each cluster with $R_{\text {proj }}<2 R_{\text {vir }}$ and $\left|V_{\mathrm{LOS}}\right|<2 \sigma_{\mathrm{LOS}}$ (the exact formulae for calculating $R_{\text {proj }}$ and $V_{\text {LOS }}$ are given in Section 3.1.2). Using these candidate member galaxies, we measured $\sigma_{\mathrm{LOS}}$ with the biweight method using up to 10 iterations and excluded galaxies with $\left|V_{\mathrm{LOS}}\right|>2 \sigma_{\mathrm{LOS}}$ in each iteration.

Then, we computed the virial masses of SDSS clusters corresponding to each $\sigma_{\mathrm{LOS}}$ by referencing the relationship between $\sigma_{\mathrm{LOS}}$ and $M_{\mathrm{vir}}$ from the YZiCS clusters (black dashed line in Figure 1(a)). The red squares in the panel represent the $M_{\text {vir }}$ and $\sigma_{\mathrm{LOS}}$ of YZiCS clusters and a clear linear correlation (with a slope of 0.37) can be seen through the black dashed line in the panel. Finally, we computed the virial radius from the virial mass based on their relation in the simulations. Thus, we argue that, in both the YZiCS and SDSS clusters, we can obtain similar normalization factors, i.e., clusters (whether observed or simulated) with a given velocity dispersion will have a comparable virial radius and mass. 


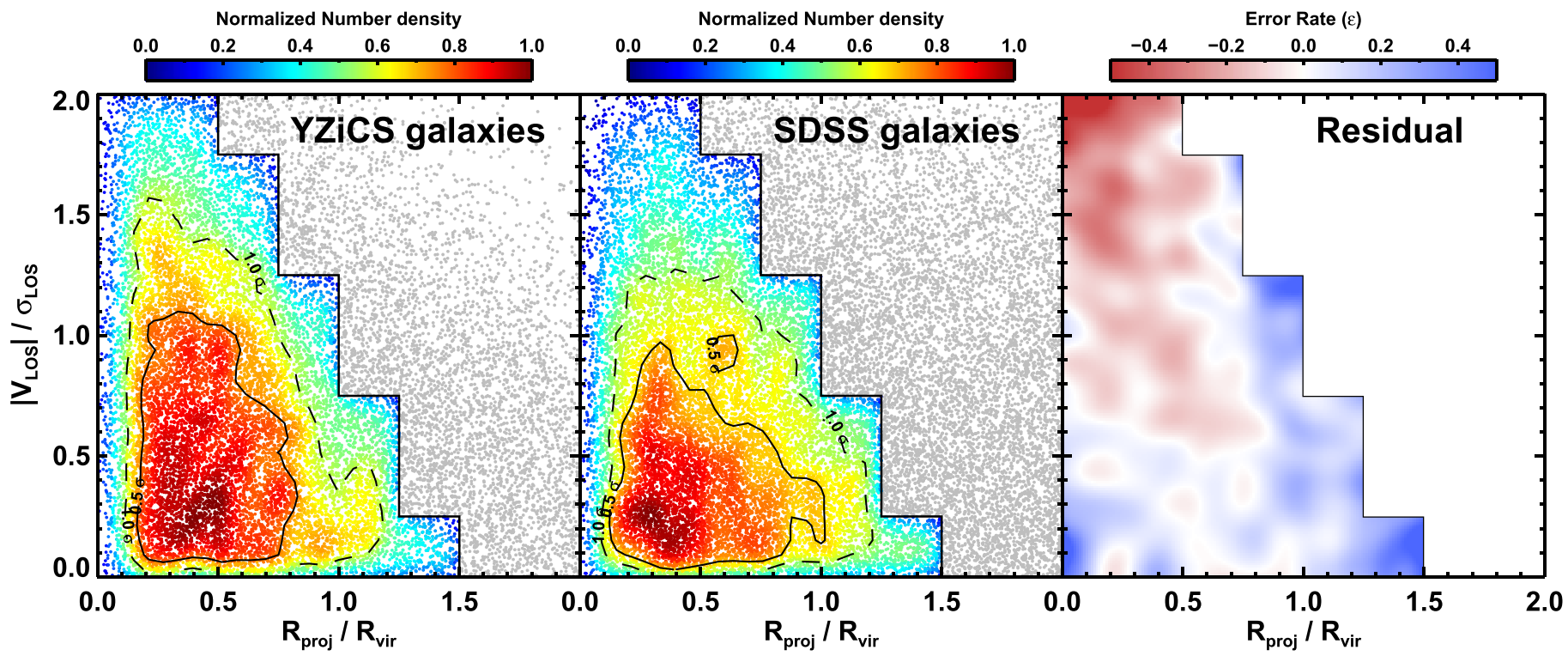

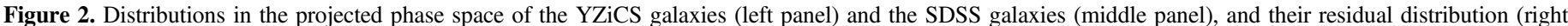

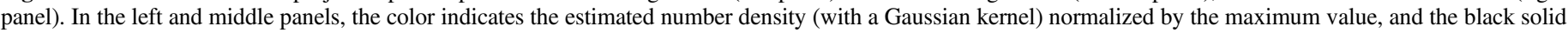

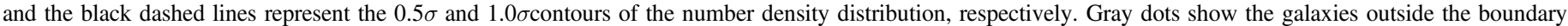

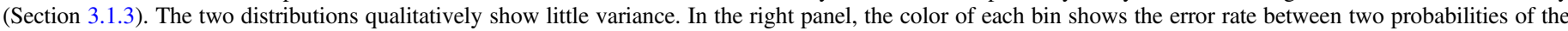

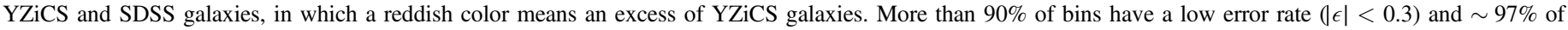
galaxies lie in these low error rate bins.

\section{TSI and SFR on the Phase-space Plane}

In this section, we will introduce how we constructed the distributions of galaxies in the projected phase space (Section 3.1), and how we derived the density function of galaxies' properties at a given position in the projected phase space (Sections 3.2 and 3.3). We connected the TSI of YZiCS galaxies with the SFRs of SDSS galaxies by the abundance matching of the two density functions at each location in the projected phase space to derive the relationship between the observed SFR at $z \sim 0$ and the TSI of cluster galaxies.

\subsection{Phase-space Distribution of Galaxies}

In this subsection, we explain how we compared the YZiCS galaxies with the SDSS sample by projecting the coordinate and introducing a flux limit $\left(m_{r}<17.77\right)$, and how we defined the phase-space coordinates of both the YZiCS and SDSS samples.

\subsubsection{Distribution of YZiCS Galaxies}

For each YZiCS cluster, we used 10 outputs with different redshifts to account for the flux limitation in observed galaxies. The choice of redshift is based on the redshift distribution of the SDSS clusters. For each YZiCS cluster, we assigned an $r$-band absolute magnitude $\left(M_{r}\right)$ to each member galaxy using their stellar mass, based on the $M_{r}$ versus $M_{*}$ relation derived from the SDSS galaxy sample, including a random scatter of 0.3 in magnitude units. Then, we computed the $r$-band apparent magnitude $\left(m_{r}\right)$ of member galaxies at the redshift of their host clusters and removed member galaxies with $m_{r}>17.77$.

To project the 3D positions of the YZiCS galaxies into a $2 \mathrm{D}$ space, we randomly selected 100 lines of sight inside the simulation box and computed the projected coordinates of each galaxy along every line of sight:

$$
R_{\text {proj }}=\left|\boldsymbol{r}_{3 \mathrm{D}}-\left(\hat{l} \cdot \boldsymbol{r}_{3 \mathrm{D}}\right) \hat{l}\right|
$$

and

$$
V_{\mathrm{LOS}}= \pm\left|\left(\boldsymbol{v}_{3 \mathrm{D}} \cdot \hat{l}\right) \hat{l}+H_{0}\left(\hat{l} \cdot \boldsymbol{r}_{3 \mathrm{D}}\right) \hat{l}\right|,
$$

where $r_{3 \mathrm{D}}$ and $\boldsymbol{v}_{3 \mathrm{D}}$ are the $3 \mathrm{D}$ clustocentric coordinates of a galaxy, $\hat{l}$ is a unit vector along the line of sight, and the sign of $V_{\mathrm{LOS}}$ is determined by the angle between $\boldsymbol{v}$ and $\hat{l}$. Thus, using the projected coordinates normalized by $R_{\mathrm{vir}}$ and $\sigma_{\mathrm{LOS}}$, the projected phase-space coordinates are derived. Similar to the SDSS clusters, $\sigma_{\mathrm{LOS}}$ in each output is computed using the galaxies with $R_{\text {proj }}<2 R_{\text {vir }}$ by the biweight method (Section 2.2.2). In summary, we used 15,000 different outputs from the 15 YZiCS clusters, in which each cluster had 1000 different outputs (10 snapshots with different redshift by 100 different lines of sight). Note that there are 2,749,945 galaxies in the total output and 1,413,860 galaxies with $m_{r}<17.77$ and $M_{*}>10^{9.5} M_{\odot}$.

The phase-space distribution of the YZiCS galaxies is shown in the left panel of Figure 2 with contours at the $0.5 \sigma$ and $1.0 \sigma$ level in the number density distribution. In the panel, we show the 15,643 galaxies randomly selected ( 1 out of every 70 ) to have a comparable number to the SDSS sample. The color code indicates the number density, normalized by the maximum value so as to easily compare with the distribution of the SDSS galaxies. The boundary is selected such that it can control the impact from foreground and background galaxies that are projected onto the clusters along our line of sight, known as "interlopers," and we explain how this boundary was selected in Section 3.1.3.

\subsubsection{Distribution of SDSS Galaxies}

We computed the projected phase-space coordinates of the SDSS galaxies with respect to the luminosity-weighted cluster 
centers:

$$
R_{\text {proj }}=d_{\mathrm{A}} \Delta \theta
$$

and

$$
V_{\mathrm{LOS}}=\frac{c\left(z_{\mathrm{m}}-z_{\mathrm{c}}\right)}{1+z_{\mathrm{c}}}
$$

where $d_{\mathrm{A}}$ is measured as the angular diameter distance, and $z_{\mathrm{c}}$ and $z_{\mathrm{m}}$ are the redshifts of the cluster and member galaxy, respectively. If a galaxy has multiple clusters in the vicinity $\left(R_{\text {proj }}<2 R_{\text {vir }}\right.$ ), we assign it to the most massive one. The middle panel of Figure 2 shows the phase-space distribution of the SDSS galaxies with the same format as in the left panel after applying the same stellar mass cut as that for the YZiCS galaxies.

By naked eye, we can see that the two distributions are similar in projected phase space, and the $0.5 \sigma$ and $1.0 \sigma$ contour lines of each density distribution match the overall shape and location.

In a more quantitative way, we computed the error rate $(\epsilon)$ between the two normalized densities:

$$
\epsilon=\frac{P_{\mathrm{obs}}-P_{\mathrm{sim}}}{P_{\mathrm{sim}}} .
$$

The right panel of Figure 2 shows the error rate at each location in which a reddish color indicates there exist more YZiCS galaxies than SDSS ones (see Figure 4 in Oman \& Hudson 2016 for a similar example). Note that $\geqslant 90 \%$ of sampled bins in the panel have $|\epsilon|<0.30$ and the mean $|\epsilon|$ is 0.13 . The presence of interlopers in the SDSS samples could induce a larger deviation at small radii and near the boundary. In any case, we confirm that these pixels have negligible effects on the final results because they occupy only a small fraction of pixels. We therefore emphasize that the phase-space distributions of the YZiCS and SDSS galaxies are clearly similar, which indicates that the accretion history of the satellite galaxies in both samples would not be significantly different.

\subsubsection{Boundary of Projected Phase Space}

In this study, we defined interlopers as galaxies that lie within the region of the projected phase space of interest $\left(R_{\text {proj }}<2 R_{\text {vir }}\right.$ and $\left.\left|V_{\mathrm{LOS}}\right|<2 \sigma_{\mathrm{LOS}}\right)$ but are actually located outside the cluster $\left(\left|\boldsymbol{r}_{3 \mathrm{D}}\right|>2 R_{\mathrm{vir}}\right)$. For the redshift range of our investigation, most interlopers are expected to be within tens of megaparsecs from the clusters considering the magnitude of the Hubble flow (see also Haines et al. 2015). The interlopers are most likely to be observed in the area of projected phase space with large values of $R_{\text {proj }}$ and $\left|V_{\text {LOS }}\right|$ (Haines et al. 2015; Oman $\&$ Hudson 2016). However, compared to the observed SDSS clusters, the YZiCS clusters inherently have a smaller fraction of interlopers because they are zoomed-in simulations. Therefore, in projected phase space, we considered only those galaxies within a limited boundary, where the presence of interlopers is expected to be minimal.

Based on the result of Oman \& Hudson (2016), we decided to use a straight-line boundary of the projected phase space, wherein the line $\left|V_{\mathrm{LOS}}\right| / \sigma_{3 \mathrm{D}}=-(4 / 3) R_{\mathrm{proj}} / R_{\mathrm{vir}}+2$ was a good demarcation of the interloper population. Because we used $\sigma_{\mathrm{LOS}}$ instead of a 3D one, the slope of the demarcation line roughly becomes -2 in our study. Then, we constructed a
1D probability density function $(f(k))$ from the 2D density functions in the projected phase space using the parameterization of $k=2 R_{\text {proj }} / R_{\text {vir }}+\left|V_{\mathrm{LOS}}\right| / \sigma_{\mathrm{LOS}}$. By varying the zero point of the boundary line, we conducted a KolmogorovSmirnov test and confirmed that $2 R_{\text {proj }} / R_{\text {vir }}+\left|V_{\text {LOS }}\right| / \sigma_{\text {LOS }}=3$ is the best choice of the boundary line with $95 \%$ significance level, assuming that two samples have the same accretion histories.

\subsection{TSI in Phase Space}

In this subsection, we will describe how we constructed the density function of the TSI of the YZiCS galaxies at each location in the projected phase space. Throughout this study, the TSI of a YZiCS galaxy is referred to as the time since the moment of infall (the first arrival at 1.5 virial radii) until $z=0.08$, which is the median redshift of the SDSS galaxies. The choice of 1.5 virial radii for the arrival boundary is based on the fact that, at distances beyond the virial radius of their clusters, infalling galaxies begin to show indications of quenching (see Haines et al. 2015; Jung et al. 2018). For reference, recent infalling galaxies in YZiCS took about $0.79_{-0.15}^{+0.16}$ Gyr to reach 1 virial radius from 1.5 virial radii, and the value decreased with increasing redshift.

We initially used the TSI data in the multiple cluster outputs (see Section 3.1.1). For galaxies that have not begun infalling and so do not have a TSI yet, we empirically derived the expected time to infall (TTI) and assign a negative value of TSI (see Section 3.2.1). In the next step, we split the projected phase space into pixels. A square pixel with grid size of 0.25 on both axes $\left(R_{\text {proj }} / R_{\text {vir }}\right.$ and $\left.\left|V_{\text {LOS }}\right| / \sigma_{\text {LOS }}\right)$ is selected to both obtain a sufficient number of pixels and avoid pixels with a low number of galaxies. We confirmed that our results remained stable under different choices of pixel size (from 0.1 to 0.3 ). For every pixel in phase space, we constructed a density function of the TSI of the galaxies inside the pixel and measured the first decile to the ninth decile of the density function (Section 3.2.3). The deciles and the measurement errors in each pixel are calculated using a bootstrap analysis, in which we resampled 1000 times, allowing replacement.

\subsubsection{TTI}

Prior to establishing the density function of the TSI, we focused on the presence of a subpopulation of interlopers, known as first infallers. We define first infallers as having $\left|\boldsymbol{r}_{3 \mathrm{D}}\right|<3 R_{\mathrm{vir}}$, but having not started infalling; thus, these objects do not have a defined TSI value yet but may appear in our projected phase-space diagram. Because most first infallers are not indeed far from host clusters, they will eventually enter the host clusters within a few gigayears. Thus, to handle such objects in the density function of the TSI, we attempted to estimate their expected TTI, i.e., the time to go from their current position to 1.5 virial radii, and we provided them with negative values of TSI, which are included in the TSI density function.

For deriving TTIs, we first focused on the YZiCS galaxies at $z \sim 0.5$, which have not fallen yet but eventually infall to host clusters before $z=0.08$. We then considered their TSIs as TTIs at $z \sim 0.5$ and explored the relationship between TTIs and their clustocentric distances at $z \sim 0.5$ (see Figure 3 ). In the figure, the data points show the TTIs and distances at $z \sim 0.5$ of the sample galaxies. The linear fitting of the data points and the first to the third quartile deviation are drawn as 


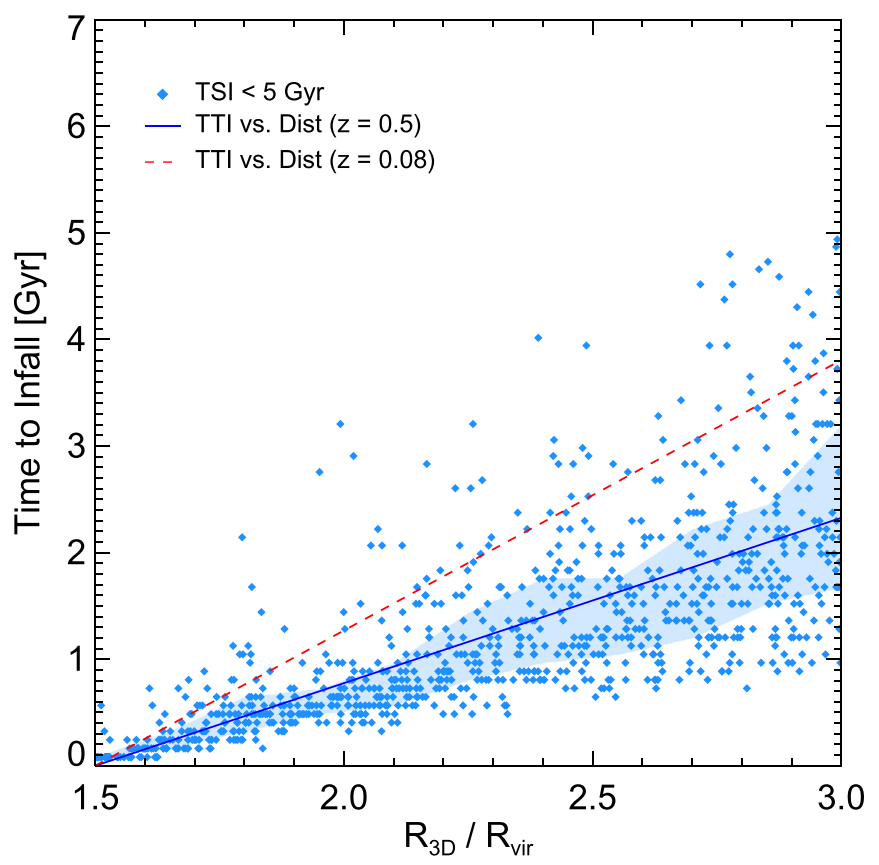

Figure 3. Relationships between the distances and TTIs of a sample of the YZiCS galaxies at $z=0.5$ (sky blue diamonds). The sample consists of YZiCS galaxies with TSI $<5 \mathrm{Gyr}$, that is, they are not falling yet, at the same redshift. The TTI of the sample galaxies is defined as the value of their TSI at $z=0.08$. The blue solid line is the linear fitting of the galaxies with the first to the third quartile deviation illustrated (shaded area). The red dashed line then indicates the same relation at $z=0.08$ while considering the redshift evolution of the crossing timescales of clusters. Based on the relationship, the TTIs of the first infallers at $z=0.08$ are empirically derived.

the blue solid line and the blue shaded area, respectively. Therefore, with the sample galaxies at $z \sim 0.5$, we roughly confirmed a linear relationship between TTIs and clustocentric distance at that epoch.

By referencing the relationship, we predicted the relation between TTIs and clustocentric distance at $z=0.08$. In this process, we considered the correction factor from the crossing time evolution of the cluster from $z=0.5$ to $z=0.08$. The predicted relationship is shown as the red dashed line in Figure 3. Based on the relationship (without considering the scatters on the relationship), we empirically estimated the TTIs of the first infallers, which allows us to include the first infallers in the TSI density function.

\subsubsection{Morphological Dependence}

In order to obtain the relationship between the SFR and TSI at $z \sim 0.08$, we used abundance matching between the density functions of the TSI (of the YZiCS galaxies) and the SFRs (of the SDSS galaxies). In Section 2.2.1, we designed the SDSS galaxy sample to be limited to disk morphologies only because we expected these to be the best tracer of environmentally affected galaxies. Likewise, during the remaining analysis, to avoid a sampling bias in the abundance matching, the YZiCS galaxies must also be restricted to disk type only. Although some previous studies using cosmological simulations have attempted to classify galaxy morphology (see, e.g., Snyder et al. 2015; Dubois et al. 2016; Rodriguez-Gomez et al. 2017), there still is controversy in the reliability of these results because of their limited spatial resolutions. The spatial resolution of today's large volume simulations $(\sim 1 \mathrm{kpc})$ is still not good enough to accurately resolve the disks in the direction out from the plane of the disk. As a result, morphology classification of our simulation, especially on disky galaxies, is extremely challenging. Therefore, we adopted an alternative approach.

We first considered the fraction of disk galaxies among the first infalling ones $\left(f_{\text {disk }}\right)$ at a given redshift. At $z=0.08$, we measured $f_{\text {disk }}=0.4$, using the galaxies located at $1.5 R_{\text {vir }}$ in our SDSS sample. We referenced the morphology-radius relation at $z \sim 1$ in Postman et al. (2005), from which we borrowed $f_{\text {disk }}=0.85$ at $0.95 R_{200}$, and then assumed that $f_{\text {disk }}=1$ at $z=3$. Second, we assumed that $f_{\text {disk }}$ linearly increases with redshift between the points. Then, we randomly assigned the morphology (either disk or not) to each YZiCS galaxy based on the derived $f_{\text {disk }}$, that is, the probability to be classified as a disk morphology is equal to $f_{\text {disk }}\left(z_{\text {inf }}\right)$, where $z_{\text {inf }}$ is the infall redshift of a YZiCS galaxy. This treatment, in principle, is based on the assumption that the timescale of morphological transition and the merger-like event rate in clusters will be long and rare, respectively (see also Wetzel et al. 2013; Lee et al. 2018; Kelkar et al. 2019). However, we note that the fractions introduced above are from different data and methodologies, so that the values in essence may have a substantial amount of scatter. Nevertheless, the difference in deciles is usually less than $1 \mathrm{Gyr}$ in most cases after considering the morphology of YZiCS galaxies, and we confirm that no matter what fraction model is chosen, our final results are hardly affected: in most cases, quenching timescales are measured within the $1 \sigma$ range of the original results.

\subsubsection{Density Functions of TSI}

By measuring the TTIs of the first infallers and considering the morphology of the YZiCS galaxies, the TSI density function of disk galaxies can be established in every pixel of the projected phase space. The left panel of Figure 4 shows the median of the TSI density function (i.e., fifth decile) in each pixel with the noted number and the color scheme. The inset panel shows an example of the density function of one pixel with the second, fifth, and eighth deciles marked (red vertical lines).

As reported in previous studies (Oman \& Hudson 2016; Rhee et al. 2017), the median of the TSI tends to be higher for inner pixels in a projected phase space. In practice, the horizontal gradient of the median is clearly visible, whereas the vertical gradient is weaker. The weak trend may be a result of projection effects, i.e., the pixels with low values of $\left|V_{\mathrm{LOS}}\right| / \sigma_{\mathrm{LOS}}$ are smeared out by galaxies with low values of TSI and high values of $\left|V_{3 \mathrm{D}}\right|$. Some YZiCS clusters have mixed distributions of TSI even in a six-dimensional phase space; therefore, a weak vertical gradient in the projected phase space is not unexpected. The dynamical history of a cluster (e.g., cluster-cluster mergers) may have a dramatic effect by mixing dynamically relaxed galaxies with the newly infalling galaxies in phase space. A much cleaner TSI density function in phase space would be derived if we could extract only very relaxed and settled clusters. However, in this work, we used all of our simulated clusters because (i) the number of simulated clusters is too small to be divided into subsamples, and (ii) it is not trivial to infer the dynamical states of the SDSS clusters.

Besides, as shown by the inset panel, some pixels have a non-unimodal TSI density function: multiple populations with different values of TSI (e.g., galaxies infalling for the first time 

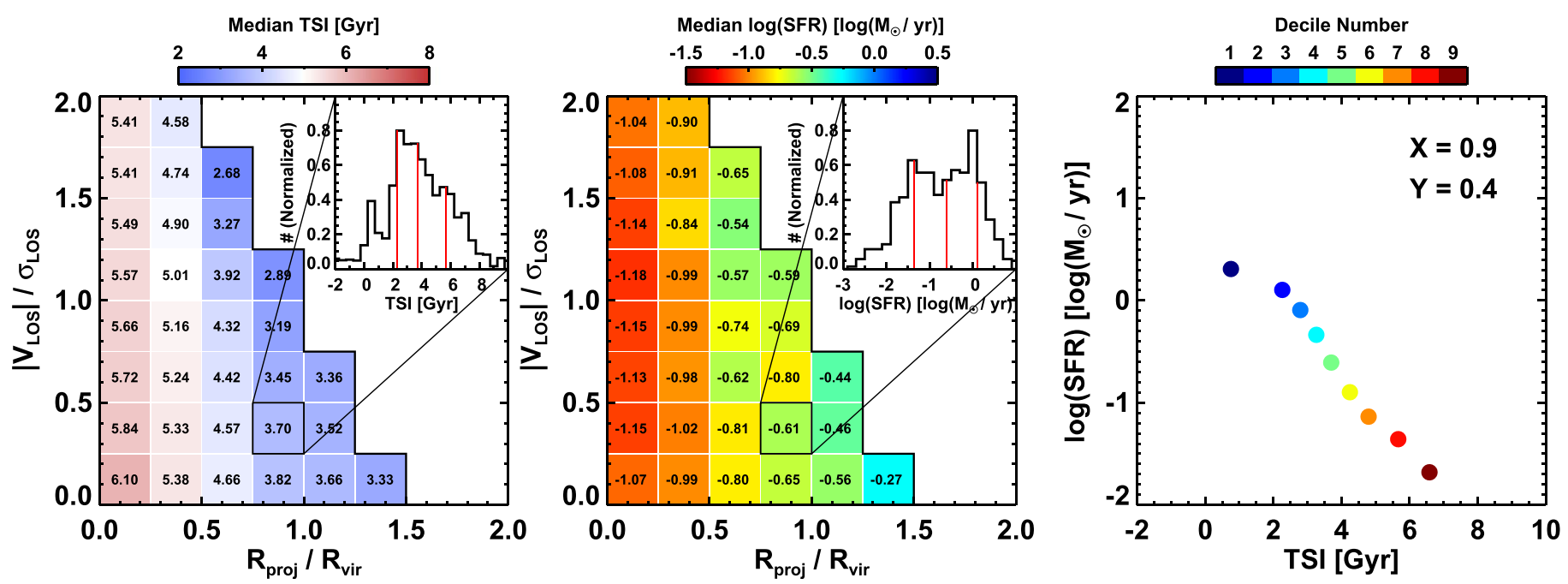

Figure 4. Left and middle panel: phase-space diagrams with the medians of the density functions of TSI (left) and SFR (middle). The median value in each pixel is noted by the color scheme. The pixel size was chosen to reach a compromise between sample size and statistical resolution (see Section 3.2). The inset in each panel is an example of a density function of a pixel, in which the second, fifth, and eighth deciles are shown with the red vertical lines. Right panel: an example of the abundance matching of the density functions. The density functions of the marked pixel in the left and middle panels are used for the abundance matching in the right panel. The color of each dot indicates the decile number of the TSI density function.

and galaxies that already passed the pericenter) can coexist at the same location in phase space. The presence of a nonunimodal feature demonstrates the richness of information stored in the TSI density function. Thus, to try to better harness this information, we used several representative values of the density function, that is, deciles. Section 4.1 gives a detailed analysis of how these deciles are used.

\subsection{SFR in Phase Space}

Analogously to Section 3.2, we created the SFR density function of SDSS disk galaxies in various locations in phase space. The middle panel of Figure 4 shows the median of the SFR density function (in logarithmic scales) in each pixel, and the inset panel illustrates the example SFR density function for the highlighted pixel with the second, fifth, and eighth deciles marked again. While the TSI density functions showed a clear gradient, most of the SFR density functions have very a low median SFR $(\log (\mathrm{SFR})<-0.5)$, that is, most of the disk galaxies (more than half) in the SDSS clusters show suppressed SFRs as previously reported (Haines et al. 2013). The inset panel shows evidence of a weak bimodal distribution of SFRs (e.g., Wetzel et al. 2013), which could be interpreted as evidence for a fast quenching scenario for satellite galaxies inside clusters. Similarly to TSI, we attempted to harness a lot of the information stored in the full SFR density function of each pixel by measuring the deciles. We used bootstrapping with 1000 resamplings to measure both the deciles and the measurement error of the deciles in each pixel.

\section{SFR-TSI Relationships}

At each pixel, we matched the TSI and SFR distribution, decile to decile, to try to deduce the connection between the two physical parameters. In this way, we explored the relationship between the TSI and SFR at $z \sim 0.08$ using our novel approach (Section 4.1). We then attempted to seek a quenching model that best reproduces the obtained SFR-TSI relationship (Section 4.2).

\subsection{SFR-TSI Relationship via Abundance Matching}

Phase-space diagrams have been used in multiple studies as a diagnostic tool to understand environmental effects. For example, several studies have adopted a phase-space tool to account for the origin of the physical state of the observed galaxies affected by the cluster environments, where each location in phase space is used to estimate the expected arrival time of the galaxies to their host clusters (e.g., Noble et al. 2013, 2016; Yoon et al. 2017; Lisker et al. 2018; Pasquali et al. 2019; Smith et al. 2019).

Despite the utility of phase-space diagrams, however, multiple studies are limited to using one specific value at each position of phase space, which may not be representative for that position. As shown in the inset panel of Figure 4, the medians of TSI or SFR have possible contamination from the other projected galaxies located in the same position of phase space. Therefore, we decided to consider the whole density function rather than a single value.

As an alternative way of considering the entire density function in a given phase-space position, we used abundance matching between the SFR and TSI density functions in each pixel, by sequentially connecting each of the deciles. The right panel of Figure 4 shows an example of the abundance matching where the density functions of the pixel highlighted in the left and middle panels are used. We assume that there is a negative correlation between the TSI and SFR, indicating that the lowest decile of the TSI density function is associated with the highest decile of the SFR density function. We can then determine the SFR-TSI relationship at $z \sim 0.08$ by repeating the abundance matching for each pixel in the projected phase space. The assumptions behind this are as follows.

1. We assume that there exists a universal trend of SF quenching for infalling disk galaxies, in which the SF quenching of an individual galaxy is largely dependent on the epoch of the arrival time to the cluster environment (see, e.g., Balogh et al. 2000; Wetzel et al. 2013; Oman \& Hudson 2016). Thus, galaxies with a similar value of TSI will have comparable SFR for a fixed galaxy mass. 
2. We postulate that each cluster galaxy has, in general, a continuously decreasing SFH with increasing cosmic time such that the SFR-TSI relationship at any cosmic epoch becomes a monotonic function. One issue that might arise is whether some galaxies undergo enhanced SF due to the compression of their ISM by ram pressure (e.g., Jaffé et al. 2018; Vulcani et al. 2018), tidal interactions, or simply a naturally bursty SFH. These factors will be addressed in Section 6.4.

Under these assumptions, the associated deciles in every pixel should follow the genuine SFR-TSI relationship. Figure 5 shows the derived relationship at $z \sim 0.08$ by matching all the deciles of the corresponding pixels, where the color of the symbol indicates the different decile number of the TSI density function. The errors are computed by combining the measurement errors of SFR and of deciles, but, in most cases, the measurement error of the SFR is the dominant one. Thus, in the plot, each pixel functionally exhibits nine points with different colors. The negative trend agrees with the expectation for more violent effects inside clusters than in the field. Furthermore, although the plot includes all pixels regardless of their location in the projected phase space, all the pixels tend to share a rather similar SFR-TSI relationship. This can be seen by observing the tendency for points with the same color (i.e., same decile number) to be smoothly connected to adjacent ones. Moreover, the relationship generally comprises two components, in which SFR is constant at the domain with low values of TSI and then immediately drops quickly after a certain value of TSI, suggesting that stronger quenching occurs inside clusters after a temporary delay. This feature is consistent with the "delayedthen-rapid" quenching model suggested by Wetzel et al. (2013), in which SF in a galaxy needs a certain amount of time after arrival to begin environmental quenching, after which the SFR is quickly reduced. Hereafter, we call the SFRTSI relationship as the "empirical" SFR-TSI relationship and will separate the relationship by host and galaxy mass in the following section.

\subsection{Quenching Model of Cluster Galaxies}

\subsubsection{Flexible Quenching Model}

Figure 5 shows that the empirical SFR-TSI relationship is a set of SFRs pertaining to galaxies with different TSI values. We emphasize that the relationship should not be considered as the SFH of a single galaxy but the synthesis set of the endpoints (i.e., at $z=0.08$ ) of individual SFHs with different values of TSI. Consequently, the challenge is to determine the SFH model that, when applied to a set of individual galaxies with different values of TSI, best reproduces the SFR-TSI relationship at $z \sim 0.08$ by tuning the quenching parameters within the model.

We adopted a flexible quenching model that allows the various aforementioned models such as rapid quenching, slow quenching, and delayed-then-rapid quenching (see Section 1 for descriptions). Along its infall trajectory, a galaxy goes through different phases of quenching that are described with different e-folding timescales. This model is similar to the one used by Wang et al. (2007; see also Wetzel et al. 2013; Contini et al. 2017; Tomczak et al. 2018), i.e., the predicted SFR of a galaxy at $z=0.08$ with a given value of TSI is as follows:

$$
\psi=\left\{\begin{array}{l}
\psi_{0} \exp \left\{\frac{-\left(t_{\text {ex situ }}+\text { TSI }\right)}{\tau_{\text {ex situ }}}\right\} \quad \text { if TSI }<t_{\mathrm{d}} \\
\psi_{0} \exp \left\{\frac{-\left(t_{\text {ex situ }}+t_{\mathrm{d}}\right)}{\tau_{\text {ex situ }}}+\frac{-\left(\text { TSI }-t_{\mathrm{d}}\right)}{\tau_{\text {cluster }}}\right\} \text { otherwise }
\end{array}\right.
$$

where TSI is the time since first infall until $z=0.08$ of a galaxy and $t_{\mathrm{ex}}$ situ is the elapsed time since $z=1$ to the infall epoch. The choice of the starting point $(z=1)$ is based on the assumption that environmental quenching was not that strong at $z \gtrsim 1$ (Gerke et al. 2007) and that most of satellite galaxies are accreted at $z<1$ (e.g., Gao et al. 2004). We confirm that the main results, except for $\tau_{\mathrm{ex} \text { situ }}$, are not sensitive to the choice of the starting redshift, as long as it is in a reasonable range (e.g., $z=1-2$ ).

In particular, the predicted SFR primarily depends on the TSI under the quenching model, and the model has four free parameters: ex situ quenching timescale $\left(\tau_{\text {ex situ }}\right)$, delay time $\left(t_{\mathrm{d}}\right)$, cluster-quenching timescale $\left(\tau_{\text {cluster }}\right)$, and the redshiftdependency index $\alpha$ of $\tau_{\text {cluster }}$. We expect some redshift dependence for the delay time and the cluster-quenching timescale because both are likely sensitive to cluster halo mass, and cluster halo mass grows with redshift (e.g., Balogh et al. 2016; Fossati et al. 2017; Nantais et al. 2017; Lemaux et al. 2019). Considering this, we adopt the redshift-dependent evolution of the two quenching parameters as follows: $t_{\text {delay }} \propto\left(1+z_{\text {inf }}\right)^{-1.5}$ (evolves like the crossing timescale; Mok et al. 2014; Muzzin et al. 2014) and $\tau_{\text {cluster }} \propto\left(1+z_{\text {inf }}\right)^{-\alpha}$ (the power as a free parameter). The above evolution models are based on the thought that the size, mass, and ICM density of clusters will be evolving with cosmic time. The redshift dependence may not be adequate for galaxies at high redshifts, however, because it is unclear whether such single power laws are physically valid over a wide redshift range (see, e.g., Balogh et al. 2016 for the case of delay time).

For example, the SFR of a galaxy is initially $\psi_{0}$ at $z=1$ and exponentially declines with the e-folding timescale (ex situ quenching timescale; $\tau_{\mathrm{ex}}$ situ $)$ before entering the cluster: "the ex situ quenching mode" (e.g., Noeske et al. 2007; Vulcani et al. 2010; Tomczak et al. 2018). After reaching the cluster boundary, it remains in the ex situ quenching mode for the delay time $\left(t_{\mathrm{d}}\right)$, i.e., "delay phase." After the delay phase, it enters the "cluster-quenching mode" in which the SFR rapidly decreases with another e-folding timescale (cluster-quenching timescale; $\left.\tau_{\text {cluster }}\right)$. The initial value of the SFR, $\psi_{0}$, is derived using the star formation MS relation at $z=1$ from star-forming galaxies (Whitaker et al. 2012):

$$
\begin{aligned}
\log \psi_{0} & =\alpha(z)\left(\log M_{*}-10.5\right)+\beta(z) \\
\alpha(z) & =0.70-0.13 z \\
\beta(z) & =0.38+1.14 z-0.19 z^{2},
\end{aligned}
$$

where we use $M_{*}$ as the mean value of galaxy masses at $z=0.08$ in each sample split by cluster mass or galaxy mass.

We consequently obtain the prediction of the SFR as a function of TSI using Equation (6) and call it the "predicted" SFR-TSI relation. We then use it for comparison with the empirical relationship shown in Figure 5. We did not distinguish between central and satellite galaxies outside the clusters (i.e., ignoring preprocessing effects). We also did not 


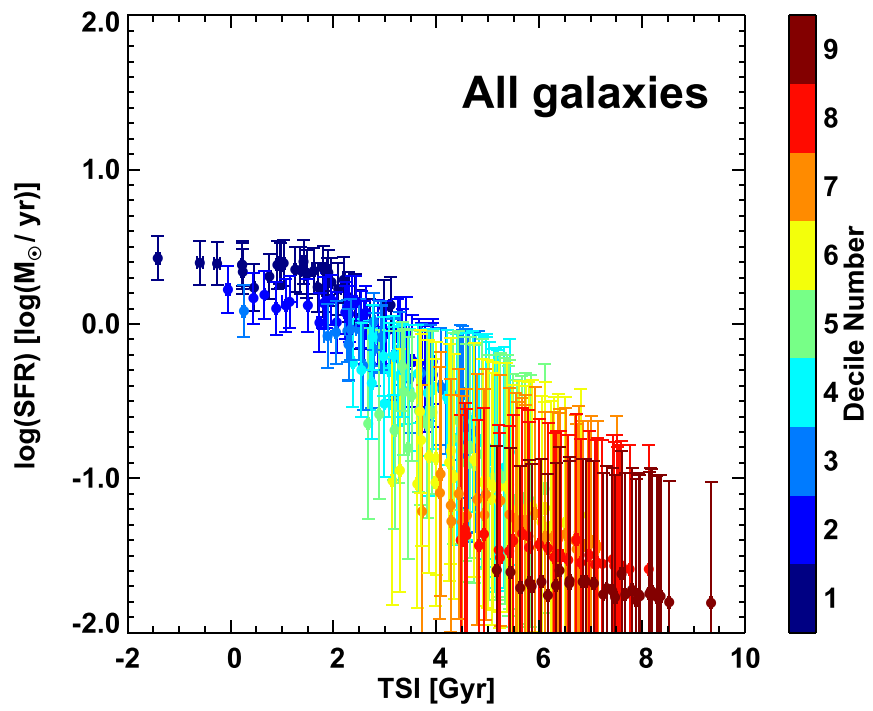

Figure 5. The SFR-TSI relationship at $z \sim 0.08$ derived by abundance matching using the deciles of each pixel of the projected phase space, thus combining all the pixels into a single figure. The color represents the decile number of each TSI density function. The error bars are estimated by combining bootstrapping errors in deriving deciles and measurement errors of TSI (or SFR). In general, there is a tendency for the SFR to remain constant after infall and then rapidly drop. This behavior is shared by the majority of the sampled pixels (see the text for details).

consider the mass growth of galaxies from $z=1$ to $z=0.08$, which indicates that our initial guess of $\psi_{0}$ can be overestimated. The overestimation of $\psi_{0}$ will result in the underestimation of the ex situ quenching timescale. For example, as an extreme case, if we assume that the stellar mass of galaxies grow by a factor of 3 from $z=1$ to $z=0.08$, which is the case for field galaxies, then the ex situ quenching timescale increases (roughly by $35 \%$ in the samples split by galaxy mass), but other quenching timescales are hardly affected.

Figure 6 shows the schematic SFHs of galaxies with different values of TSI. In panel (a), the three colored arrows indicate the SFH of a galaxy with TSI $=4 \mathrm{Gyr}$, and the gray points are the empirical SFR-TSI relationship of a sample binned by stellar mass. The galaxy first follows an exponentially decaying $\mathrm{SFH}$, the ex situ quenching mode, before entering the cluster (blue arrow). Under the delayed-then-rapid scheme, the timescale of the ex situ quenching mode remains the same beyond the arrival at $1.5 R_{\mathrm{vir}}$ for the duration of the delay time, $t_{\mathrm{d}}$ (green arrow). After the delay time, the clusterquenching mode (red arrow) begins. Panel (b) demonstrates the SFH of a galaxy with $\mathrm{TSI}=1.5 \mathrm{Gyr}$, where we assume TSI $<t_{\mathrm{d}}$. Thus, the cluster-quenching mode has not been reached in this example; therefore, this galaxy has a high value of SFR. Panel (c) is another example of a galaxy with a high value of TSI. The longer time since the delay results in a very low value of the SFR.

\subsubsection{Fitting Description}

We will now attempt to constrain the fitting parameters by comparing the empirical (Section 4.1) and predicted SFR-TSI relationships (Section 4.2.1). In order to see whether quenching depends on either stellar mass of galaxies or their host cluster mass, we divide the sample into six bins according to stellar mass and into two bins according to cluster mass. The mass range among bins is chosen to ensure robustness against low number statistics, so as to obtain sufficient pixels for each bin. For our test, we did not see a significant dependency with cluster mass (see Figure 7). But it is likely that the number of clusters in YZiCS is too few to divide the sample by cluster mass and check if there is cluster mass dependence. Therefore, in the remaining analysis, we will focus on the dependency on stellar mass.

In each binned sample, the likelihood of the predicted SFRTSI relationship for a set of quenching parameters can be written as

$$
\mathcal{L}=P\left(X_{1}, \cdots, X_{N} \mid \tau_{\text {ex situ }}, \tau_{\text {cluster }}, t_{\mathrm{d}}, \alpha\right),
$$

where $X_{i}$ is each point in the empirical SFR-TSI relationship. Originally, there were 32 pixels inside the boundary of the projected phase space (Section 3.1.3), and each pixel had nine points extracted from the density functions, that is, a total of $N=288$. However, we excluded the pixels with a small number of galaxies $\left(N_{\text {gal }}>20-40\right)$ and removed the deciles with sSFR $<10^{-11.7} \mathrm{yr}^{-1}$ considering the empirical detection limit as suggested by Salim et al. (2016).

We then assumed that each pixel is independent and identically distributed, therefore,

$$
P\left(X_{1}, \cdots, X_{N} \mid \tau_{\text {ex situ }}, \tau_{\text {cluster }}, t_{\mathrm{d}}, \alpha\right)=\prod_{i} P_{i},
$$

where $_{P i}$ is a likelihood in the $i$ th pixel. Finally, we assumed flat priors for the fitting parameters, i.e., the posterior probability for the deciles in each pixel can be represented by

$$
P_{i} \propto \exp \left\{\sum_{n} \frac{-\left(\psi_{n}-\bar{\psi}_{n}\right)^{2}}{2\left(\sigma_{\mathrm{n}, \text { boot }}^{2}+\sigma_{\mathrm{n}, \text { measure }}^{2}+\sigma_{\mathrm{n}, \text { model }}^{2}\right)}\right\},
$$

where $\psi_{n}$ and $\bar{\psi}_{n}$ are the empirical and predicted SFRs at a given value of TSI in the $i$ th pixel, respectively. The errors, $\sigma_{\mathrm{n} \text {,boot }}, \sigma_{\mathrm{n} \text {,measure }}$, and $\sigma_{\mathrm{n} \text {,model }}$, are the estimated errors of the decile $\left(\psi_{n}\right)$ measurement, SFR measurement, and model prediction $\left(\bar{\psi}_{n}\right)$, respectively. Finally, we performed the Markov Chain Monte Carlo method to constrain the parameters. In this manner, by regulating the fitting parameters, we can infer the best quenching model of cluster galaxies that the points of the empirical SFR-TSI relationship prefer. For instance, the slow quenching model (see, e.g., Haines et al. 2013) favors very small and moderate values of $t_{\mathrm{d}}$ and $\tau_{\text {cluster, }}$, respectively, while the delayed-then-rapid model requires a nonzero value of $t_{\mathrm{d}}$ and then a small value of $\tau_{\text {cluster. }}$

Figure 8 shows the result of the constrained fitting parameters in each stellar mass bin, the mass range of which is noted on the upper left in each panel. Gray dots show the SFR from multiband spectral fits and the TSI from the simulation, that is, the empirical SFR-TSI relationships (Section 4.1). The black solid line indicates the predicted SFR-TSI relationship from the best-fitting parameters. The colored arrows show the SFH of an example galaxy, with TSI $=4 \mathrm{Gyr}$, where the color scheme has the same format as Figure 6. The dashed horizontal line in the panel corresponds to $\mathrm{sSFR}=10^{-11.7} \mathrm{yr}^{-1}$ as a detection limit of SFR measurement (Salim et al. 2016). We exclude the data points with sSFR $<10^{-11.7} \mathrm{yr}^{-1}$ in the fitting process. As seen in the panels, all the predicted SFR-TSI relationships show a good level of agreement with the empirical ones. The predicted relations (black solid lines) are basically the compilation of 

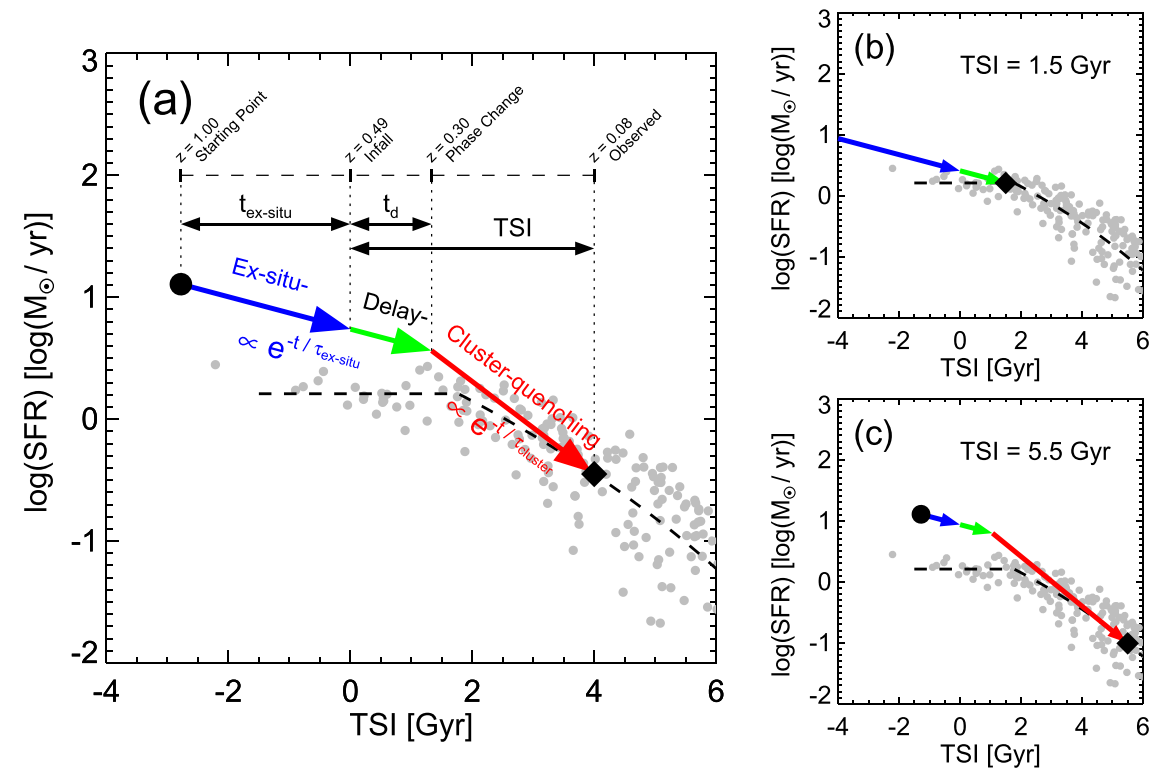

Figure 6. SFH model of galaxies with given values of TSI. Panels (a)-(c) correspond to TSI = 4 Gyr, 1.5 Gyr, and 5.5 Gyr, respectively. In each panel, the blue arrow indicates the ex situ quenching mode $\left(\propto e^{\frac{-t}{T_{\mathrm{ex}} \text { situ }}}\right)$, and the green arrow illustrates the delay phase before the sudden drop of SFR following arrival at the clusters, preserving its original evolution phase. The red arrow represents the cluster-quenching mode $\left(\propto e^{\frac{-t}{T_{\text {cluster }}}}\right)$. In Section 4.2.1, the SFH model is described in more detail; the gray dots are examples of the SFR-TSI relationship of galaxies within a mass bin of $10^{9.9-10.2} M_{\odot}$. The diamond symbol is the observed epoch $(z \sim 0.08)$. This figure shows how the value of TSI determines the observed SFR under a given quenching model.
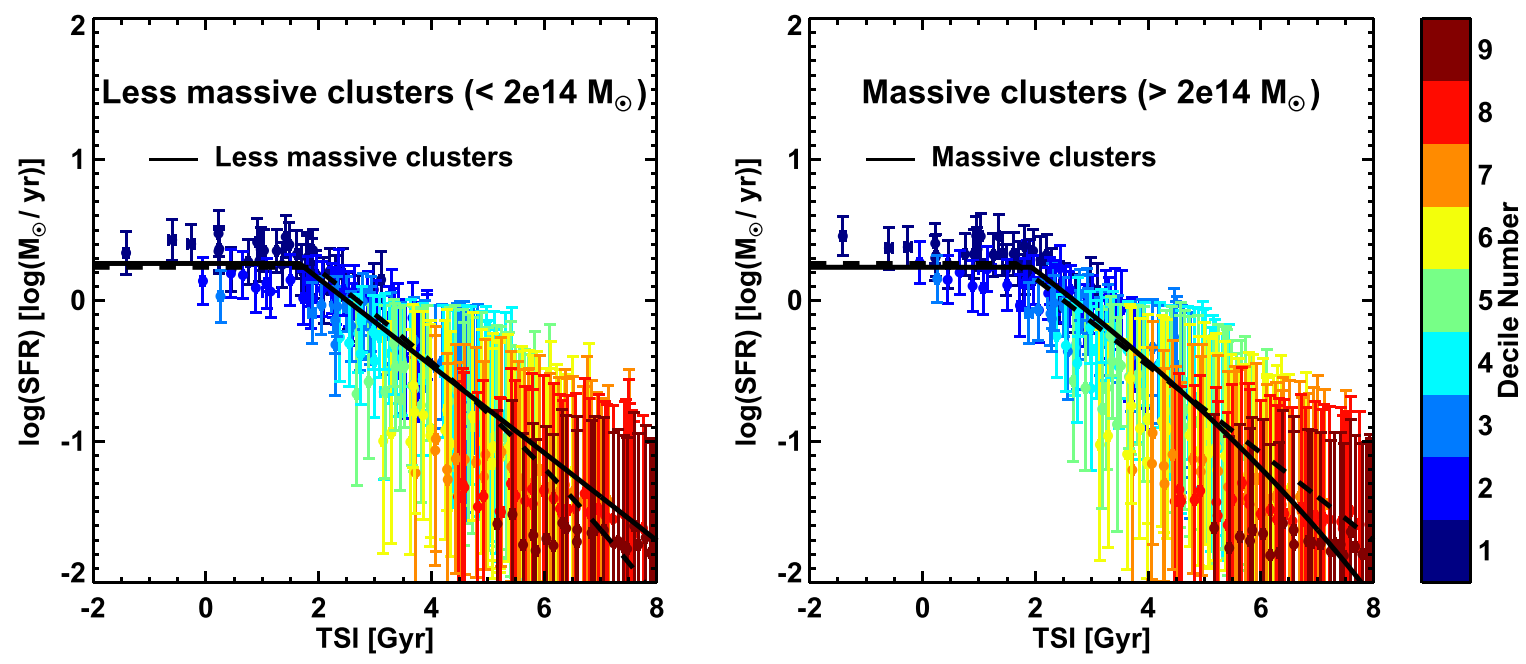

Figure 7. The SFR-TSI relationships with different cluster mass ranges. The colors have the same format as Figure 5, and the errors of each datum are shown together. The cluster mass range is written at the top of each panel. The solid line is a fitting curve (Section 4.2.2) for the corresponding mass range, and the dashed line is the fitting curve of the other sample, shown for easy comparison. The results qualitatively indicate that the host mass dependency is fairly weak (Section 4.2.2).

galaxies falling into the clusters at different epochs. When the TSI is negative or small (typically below 2 Gyr), it appears flat simply because we assumed a linear relation between cosmic time and $\log (\mathrm{SFR})$ in Equation (6).

The marginal distribution of each fitting parameter is shown in Figure 9. The first column shows the SFR-TSI distributions of galaxies with different masses. The second column shows the marginalized distribution of $\tau_{\mathrm{ex}}$ situ. It is overall well constrained with a narrow single peak. The third column shows the cluster-quenching timescale $\left(\tau_{\text {cluster }}\right)$. It is well constrained for low-mass galaxies, but poorly constrained for high-mass galaxies. This is mainly due to the fact that the more massive galaxies are more passive in terms of SF and hence go below the observational detection limit; as a result, fewer data points are available for fits. Apart from that, we find that the marginalization is reasonably satisfactory. The last column shows the redshift dependence parameter of cluster-quenching timescale $(\alpha)$. Like the cluster-quenching timescale, $\alpha$ is rather poorly constrained for high-mass galaxies for the same reason. Otherwise, the marginal distribution is consistent with no redshift dependency, that is, $\alpha \approx 0$. Degeneracy is notably strong only between $\alpha$ and $\tau_{\text {cluster }}$ because they are mutually connected: $\tau_{\text {cluster }} \propto\left(1+z_{\text {inf }}\right)^{-\alpha}$.

While we find that the marginalization is rather satisfactory by and large, we would like to remind the readers that our fitting procedure is based on a few assumptions that are loosely grounded and may affect the validity of our results to some extent. As we mentioned in Section 4.2.1, the assumptions on 

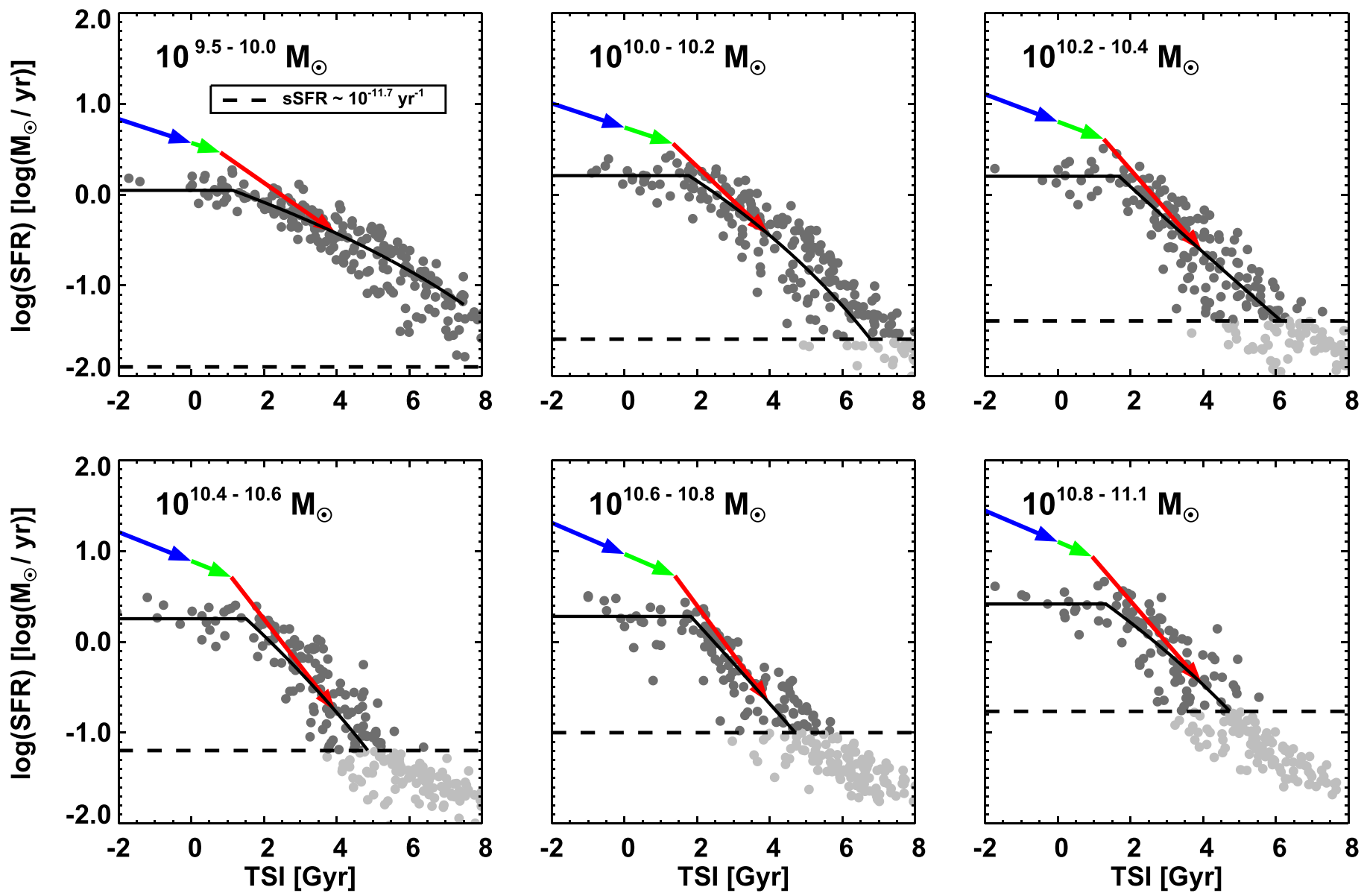

Figure 8. The SFR-TSI relationships in different stellar mass bins. Each panel has the same format as in Figure 5 and illustrates the dependency on the stellar mass bin noted in the upper-left corner of each panel. The error bar and the decile number of each symbol are removed for clarity; both are shown in Figure 9. The black solid lines represent the predicted SFR-TSI relationship based on the best-fit quenching parameters. The colored arrows show an example of the SFH for a single galaxy with TSI $=4 \mathrm{Gyr}$, in which blue, green, and red indicate the ex situ, delay-, and cluster-quenching phases, respectively. The horizontal dashed line corresponds to values of $10^{-11.7} \mathrm{yr}^{-1}$ in each panel, which is a measurement limit of the SFR from Salim et al. (2016).

the single power-law redshift dependence of delay time and cluster-quenching timescale are such examples.

\section{Results}

\subsection{Ex Situ Quenching Timescale}

Panel (a) of Figure 10 shows the constrained ex situ quenching timescale $\left(\tau_{\text {ex situ }}\right)$ as a function of stellar mass. The ex situ quenching timescale corresponds to the e-folding timescale of the SFR of cluster galaxies from our initial guess (SFR at $z=1$, from the MS relationship; Equation 7) to the period until they are accreted onto their clusters. Thus, in some respects, the ex situ quenching timescale is similar to the evolution of field galaxies while also including some effects from group preprocessing or is consistent with the e-folding timescale of galaxies in the group/filament environments (e.g., Taranu et al. 2014). For example, these timescales are generally lower than the predicted e-folding timescale of the global SFH at $z<1$ (3.9 Gyr; Madau \& Dickinson 2014), probably due to the additional effect of preprocessing. The ex situ quenching timescale has been estimated to be 2.5-3.5 Gyr from our analysis. The mass dependence is clearly visible in the sense of decreasing $\tau_{\text {ex situ }}$ with increasing mass. These results are all consistent with the earlier reports of Wetzel et al. (2013; see also Oman \& Hudson 2016). The inverse mass trend of $\tau_{\text {ex situ }}$ may be a result of the mass effect on the SFR of galaxies: that is, more massive galaxies often show lower SFRs. Considering the stellar mass trend, therefore, internal quenching processes prior to infall had an important role in massive galaxies even in dense regions. We tried to fit the result using a single power law, with the form of $\tau_{\text {ex situ }} \propto M_{*}^{\gamma}$, shown as the blue dashed line in the figure. The resultant fitting power is $\gamma=-0.45$.

We, however, note that the derived values of the ex situ quenching timescale are in principle dependent on the choice of the initial redshift. For example, we attempted to derive ex situ quenching timescale from the MS relationship at different initial redshift values $(1<z<2)$. When we try a larger value of the initial redshift, a smaller quenching timescale is obtained. Our standard choice was $z=1$, and the try with the MS relation at $z=2$ (Equation (7)) gave the largest difference yielding $\gamma=-0.66$. Yet, the general mass trend remains the same. Trying different versions of MS relations (e.g., Tomczak et al. 2016) led to a similar result with no significant difference in the mass trend.

\subsection{Delay Time}

The second quenching parameter to consider is delay time, the time from the infall to the beginning of the clusterquenching mode (Figure 10, panel (b)). In our analysis, the 

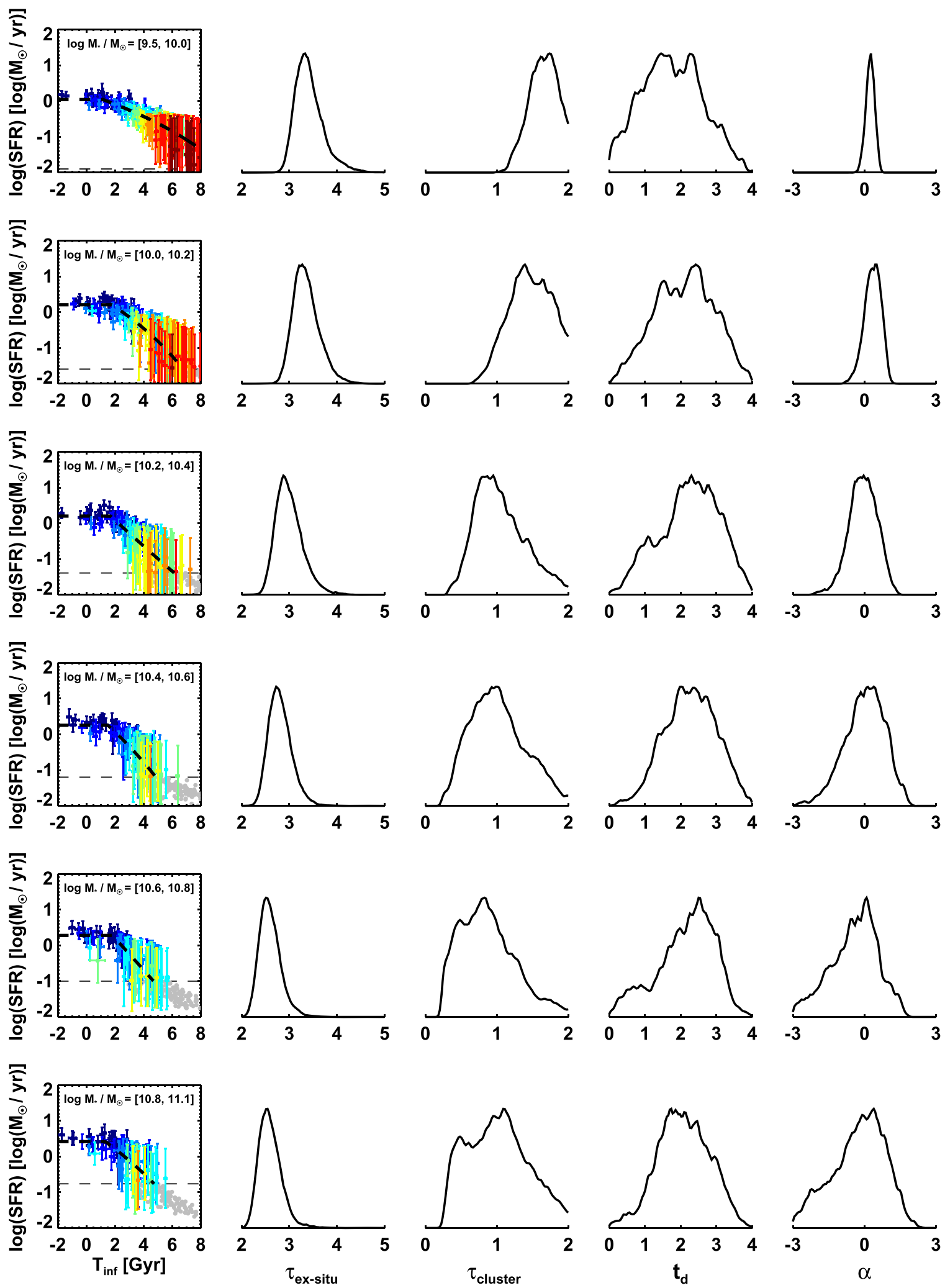

Figure 9. Marginal distribution of each quenching parameter. First column: the SFR-TSI relationships shown in Figure 8 with the error bars shown. Colors indicate the decile numbers illustrated in Figure 5. Second to the last column: marginal distribution of the quenching parameters.

delay time is measured to be $1.8-2.6 \mathrm{Gyr}$, and we note that these values are estimated at $z \sim 0.08$ considering the redshift dependence of delay time. The values are approximately constant in all mass ranges and surprisingly comparable with the typical crossing time of clusters (green area), thus showing a good level of agreement with previous studies (Mok et al. 2014; Tal et al. 2014; Fossati et al. 2017; Lemaux et al. 2019). We measured the crossing time as the mean of the times for the first infalling satellite galaxies to travel from $1.5 R_{\mathrm{vir}}$ to the pericenter in the YZiCS clusters. 

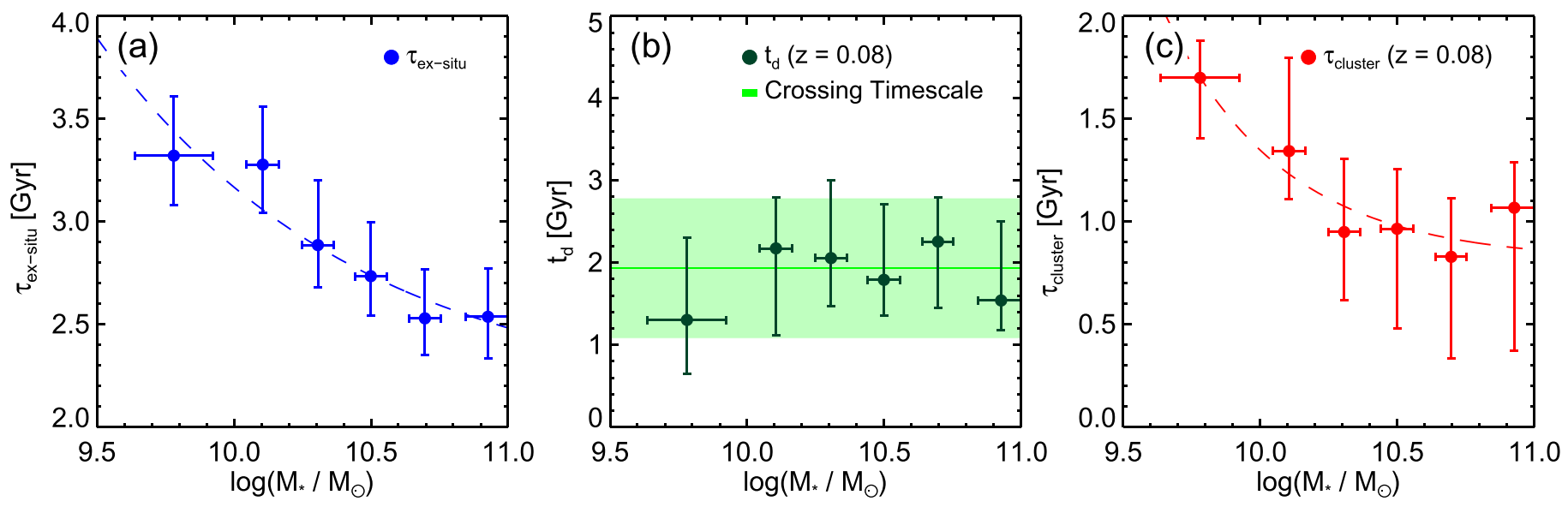

Figure 10. Derived quenching parameters as a function of stellar mass. From panels (a)-(c), the ex situ quenching timescale, delay time, and cluster-quenching timescale are shown, respectively. The ex situ quenching timescale is the e-folding timescale at $z=1$, at which the derived SFH falls. Due to redshift-dependent factors, delay times at $z=0.08$ and cluster-quenching timescales at $z=0.08$ are presented. In panels (a) and (c), the dashed lines indicate the fitting curve with a simple power law $\left(\propto M^{\alpha}\right)$. In panel (b), the green shaded region represents the crossing time of the YZiCS galaxies with the error in dex. The errors of all data points are defined as the $1 \sigma$ confidence interval.

Considering the similarity between the delay time and the crossing time, we may naturally argue that the first pericenter passage can be the end of the delay phase (see, e.g., Mok et al. 2014; Balogh et al. 2016; Paccagnella et al. 2016; Foltz et al. 2018; Lotz et al. 2019). Gas removal mechanism during the delay phase, if it is really in action, may be moderate and biased, in the sense that it is not so extremely violent to remove all of the gas in a short period of time but instead more effective on the less tightly bound hot and neutral gas than on molecular gas (e.g., Casoli et al. 1998). Such a moderate process would remove some gas from galaxies without affecting SFRs much. This has been observed in some spiral galaxies in the Virgo cluster (Lee et al. 2017). Star formation is largely unaffected during the delay phase, hence the name "delay," but after the first pericenter pass, even molecular gas might be removed, affecting SF dramatically.

\subsection{Cluster-quenching Timescale}

The cluster-quenching timescale $\left(\tau_{\text {cluster }}\right)$ is an e-folding timescale in the SFR evolution during the cluster-quenching mode and presented as a function of stellar mass in Figure 10(c). Accepting the possibility of redshift dependence of the cluster-quenching timescale, we allow the following variation and try to find the best-fitting value of the dependence through our fitting exercise: $\tau_{\text {cluster }} \propto\left(1+z_{\text {inf }}\right)^{-\alpha}$. The timescale presented in this figure has been measured at $z=0.08$ to be consistent with the median redshift of the SDSS galaxy sample.

The cluster-quenching timescales are short $(0.7-1.5 \mathrm{Gyr})$ compared to the other two timescales discussed above, indicating that cluster galaxies quickly become passive as soon as the cluster-quenching mode operates. This is consistent with previous findings, both observational and theoretical (Haines et al. 2013; Wetzel et al. 2013; Mok et al. 2014; Muzzin et al. 2014; Balogh et al. 2016; Boselli et al. 2016; Fossati et al. 2017, 2018; Jung et al. 2018; Roberts et al. 2019). The most likely candidate process for such a fast quenching is the direct gas removal process, i.e., ram pressure stripping (e.g., Boselli et al. 2016; Fossati et al. 2018; Jung et al. 2018).

The cluster-quenching timescale shows a mass dependence. At high masses $\left(M_{*}>10^{10.2} M_{\odot}\right)$, the cluster-quenching timescale shows roughly constant values. It may be a result of the competition between internal and external effects. On the one hand, more massive galaxies can better resist the direct gasstripping process (e.g., ram pressure), thanks to their stronger gravitational restoring force (e.g., Pasquali et al. 2019; Smith et al. 2019). On the other hand, more massive galaxies tend to be more (partially) self-quenched even before entering clusters, which makes the ram pressure stripping more effective (Jung et al. 2018).

The values of the cluster-quenching timescales of low-mass $\left(M_{*}<M_{\odot}^{10.2}\right)$ galaxies may appear too large compared to the simple expectation based on the role of restoring force against a direct gas removal process. This trend has also been reported by previous studies in the sense that galaxies become most resistant against environmental quenching at $10^{9}-10^{10} M_{\odot}$ (e.g., Wheeler et al. 2014; Mistani et al. 2016; Oman \& Hudson 2016). To understand this, many studies focused on the fact that lessmassive galaxies have a larger gas fraction with lower SF efficiency (slow starvation; Wheeler et al. 2014). In addition, if inside-out mass quenching is more pronounced in more massive galaxies, it is possible that low-mass galaxies have a larger gas fraction at the disk center than massive galaxies do. This is because mass quenching can cause the gas density to be more centrally peaked (e.g., Tacchella et al. 2016). The centrally peaked gas is probably more resistant to ram pressure, because it feels a greater restoring force. As a result, lower mass galaxies might retain their central gas for a longer period of time, allowing a longer cluster-quenching timescale.

We determined that $\tau_{\text {cluster }} \propto M_{*}^{-1.00}$ yields the best-fitting power law (the red dashed line), which suggests a stronger stellar mass dependency than $\tau_{\text {ex situ }}(-0.45)$. Perhaps, through a complex combination of different competing effects, mass has turned out to be an important factor, even for cluster environmental quenching.

\section{Discussion}

\subsection{Summary of the Quenching Timescales}

In Section 5, we presented how different quenching stages and their associated timescales have been measured. The measured values of quenching parameters in each stellar mass 
Table 1

Quenching Parameters

\begin{tabular}{ccccccc}
\hline \hline $\log \left(M_{*} / M_{\odot}\right)^{\mathrm{a}}$ & $t_{\mathrm{Q}}(\mathrm{Gyr})^{\mathrm{b}}$ & $t_{\mathrm{Q}}(\mathrm{Gyr})^{\mathrm{c}}$ & $\tau_{\text {ex situ }}(\mathrm{Gyr})^{\mathrm{d}}$ & $\tau_{\text {cluster }, 0}(\mathrm{Gyr})^{\mathrm{e}}$ & $\alpha^{\mathrm{f}}$ & ${ }_{\mathrm{d}, 0}^{\mathrm{g}}(\mathrm{Gyr})$ \\
\hline $9.5-10.0$ & 7.45 & 5.45 & $3.32_{-0.24}^{+0.29}$ & $1.73_{-0.30}^{+0.18}$ & $0.27_{-0.20}^{+0.17}$ & $1.46_{-0.73}^{+1.12}$ \\
$10.0-10.2$ & 5.20 & 4.25 & $3.28_{-0.23}^{+0.28}$ & $1.39_{-0.24}^{+0.47}$ & $0.49_{-0.46}^{+0.25}$ & $2.43_{-1.19}^{+0.69}$ \\
$10.2-10.4$ & 4.15 & 3.38 & $2.89_{-0.20}^{+0.32}$ & $0.95_{-0.35}^{+0.35}$ & $-0.01_{-0.61}^{+0.58}$ & $2.30_{-0.65}^{+1.06}$ \\
$10.4-10.6$ & 3.38 & 2.91 & $2.73_{-0.19}^{+0.26}$ & $1.00_{-0.30}^{+0.30}$ & $0.41_{-1.03}^{+0.66}$ & $2.00_{-0.48}^{+1.03}$ \\
$10.6-10.8$ & 3.14 & 2.87 & $2.53_{-0.18}^{+0.14}$ & $0.83_{-0.49}^{+0.28}$ & $0.09_{-1.35}^{+0.60}$ & $2.52_{-0.90}^{+0.60}$ \\
$10.8-11.1$ & 2.86 & 2.78 & $2.54_{-0.20}^{+0.23}$ & $1.10_{-0.71}^{+0.22}$ & $0.39_{-1.41}^{+0.79}$ & 27 \\
\hline
\end{tabular}

Notes.

a Stellar mass range.

${ }^{\mathrm{b}}$ Quenching times following the criterion of Wetzel et al. (2013) as discussed in Section 6.2.

${ }^{c}$ Quenching times following the criterion of Oman \& Hudson (2016) as discussed in Section 6.2.

d Ex situ quenching timescale.

e Cluster-quenching timescale predicted at $z=0$.

${ }^{\mathrm{f}}$ Redshift-dependent factor of the cluster-quenching timescale: $\tau_{\text {cluster }}\left(z_{\text {inf }}\right)=\tau_{\text {cluster, } 0}\left(1+z_{\text {inf }}\right)^{-\alpha}$.

${ }^{\mathrm{g}}$ Delay time predicted at $z=0: t_{\mathrm{d}}\left(z_{\text {inf }}\right)=t_{\mathrm{d}, 0}\left(1+z_{\text {inf }}\right)^{-1.5}$.

${ }^{\mathrm{h}}$ Number of pixels used in the processes of fitting.

bin are listed in Table 1. We here describe the results in terms of TSI, i.e., the stage of arrival history into the cluster.

(i) When it comes to first infalling galaxies (TSI $<0$ Gyr, that is, when galaxies are yet to arrive at the cluster), massive galaxies have shorter ex situ quenching timescales (Figure 10(a)) in their SFR evolution. The trend of the ex situ quenching timescale against stellar mass may follow the prediction of mass quenching that stellar mass can be an indicator of the strength of the quenching (see also Peng et al. 2010). Shorter ex situ quenching timescales for more massive galaxies cause them to have lower values of sSFR at the moment of infall (Figure 8), such that mass quenching prior to infall plays a significant role in producing passive galaxies (i.e., red sequence) inside clusters (e.g., Lidman et al. 2008; Kimm et al. 2009).

(ii) In our SFH model, galaxies with $\mathrm{TSI} \lesssim t_{\mathrm{d}}$ (i.e., in the delay phase) have the same evolutionary path of SFR as the galaxies that are outside the clusters. Combined with the consistency of delay times with the crossing times $\left(t_{\mathrm{d}} \sim t_{\text {cross }}\right.$; Section 5.2), we deduce that SFRs of first infalling galaxies are gently quenched by clusters until the first pericenter passage. The most important factor controlling such a gentle mode of quenching is how the gas reservoir is maintained without affecting the SFR much (e.g., Taranu et al. 2014). In the process of gentle quenching, the stripping of neutral and hot gas, rather than molecular gas, may happen more easily (e.g., Casoli et al. 1998; Lee et al. 2017). In addition, once inside clusters, galaxy halos are unlikely to accrete gas from outside any longer. These two effects combined may be the main reason for the gentle quenching (Section 5.2).

(iii) For TSI $\gtrsim t_{\mathrm{d}}$ (i.e., beyond the first pericenter pass), however, when a strong ram pressure is thought to begin to work on galaxies (Jung et al. 2018), our analysis demonstrates that galaxies are likely to be quenched on very short timescales (Figure 10(c)). This is inferred by the rapid drop of the SFR at TSI $\gtrsim 2$ Gyr in Figure 8. A mass dependence of cluster-quenching timescale was visible, and the particularly larger values of low-mass galaxies were noteworthy. This may be a result of the higher gas contents and perhaps steeper radial gas density profiles of

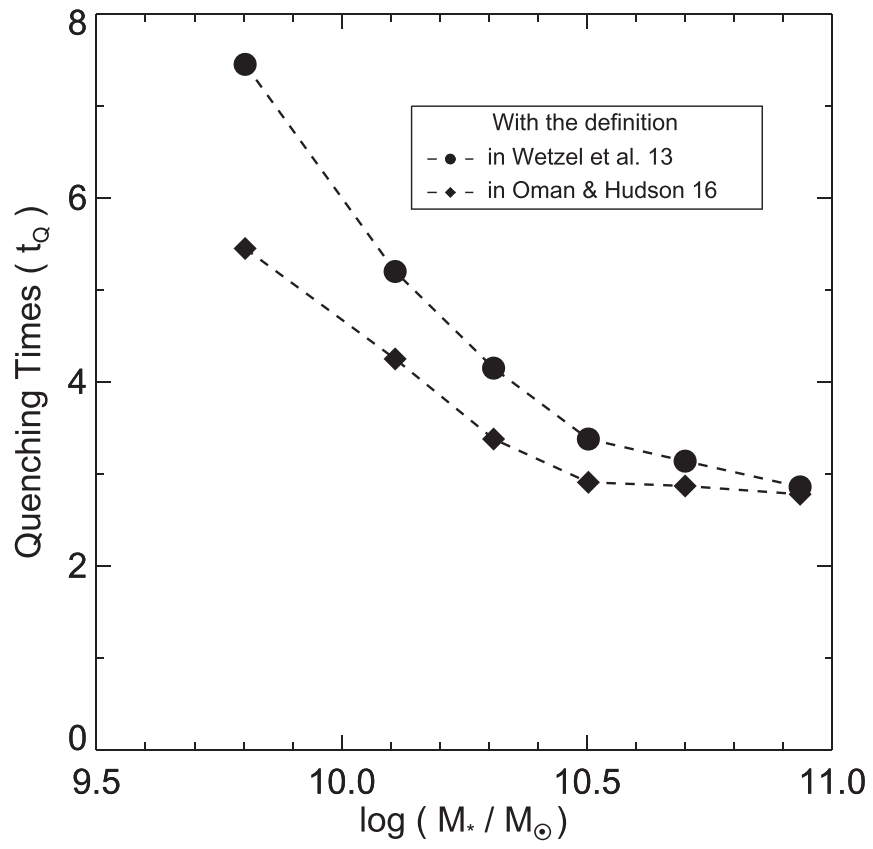

Figure 11. Derived quenching times as a function of stellar mass. Quenching times are defined as the TSI of galaxies becoming passive at $z=0.08$. Passive galaxies are separated using two definitions that were described in Wetzel et al. (2013, black dashed line) and Oman \& Hudson (2016, gray dashed line; see the text for the exact description of definitions).

low-mass galaxies, both of which help low-mass galaxies maintain their SF for a longer period of time.

\subsection{Quenching Time}

How long does it take for a star-forming galaxy to be quenched once it enters a cluster? This "quenching time" (or, more specifically, cluster-quenching time) has been used as a standard index for inferring the dominant quenching process. The quenching time is obviously a result of the three timescales we have derived in our study combined. We can also measure the "present-day quenching time" by measuring the times since infall of the galaxies that have just reached the criterion for SF quenching say at $z=0.08$. Figure 11 shows the result with 
respect to the stellar mass of galaxies. When a quenching condition of sSFR $<10^{-11} \mathrm{yr}^{-1}$ is used, the typical quenching time (top curve) is roughly $3-7 \mathrm{Gyr}$ with a mild mass dependence, which is expected considering the previous findings on the three timescales above. This is qualitatively consistent with the result of Wetzel et al. (2013). Our values are very close to their result for the four massive bins, while the two lowest mass bins are higher in our estimates. It should, however, be noted that a direct comparison is not possible because there is difference in the measurement technique for TSI as well as SFRs.

If we use a different cut for quenching, e.g., $\log \left(\mathrm{sSFR} / \mathrm{yr}^{-1}\right)<-\frac{\log \left(M_{*} / M_{\odot}\right)}{25}-6.6$ following Oman \& Hudson (2016), we find slightly lower values (lower curve), which is again consistent with the result of Oman \& Hudson (2016), if we use the same definition for TSI. In conclusion, the three timescales we derived in our investigation yield a result that is consistent with previous and independent findings. We provide the quenching times discussed in this subsection in Table 1 as well.

\subsection{Effects from Preprocessing}

In Section 4.1, we made the assumption that there is a universal way of quenching cluster galaxies, and each cluster galaxy has a continuous decline in its SFH once inside its cluster. Preprocessing, however, could potentially lower SFRs before galaxies reach the cluster and also during the delay phase, and hence, induce a large spread in the vertical direction of an SFR-TSI relationship within TSI $\lesssim 2$ Gyr (Figure 8). Indeed, we may see a hint of this in action, because the derived values of the ex situ quenching timescale $\left(\tau_{\text {ex situ }}\right)$ are lower than the prediction from the e-folding timescale in the global SFH (3.9 Gyr; Madau \& Dickinson 2014). The effect of preprocessing is in principle implicitly included in our measurement for ex situ quenching timescale, but it would be interesting to repeat our exercise on group environments separately to constrain the quenching history inside small halos.

\subsection{Enhanced SF inside Clusters}

Galaxies may temporarily experience enhanced SF while undergoing ram pressure stripping (Bekki 2014; Steinhauser et al. 2016; Vulcani et al. 2018), which is missing in our analysis. The enhancement, however, is considered to last a short period of time and thus, rare. We conducted a test to examine how sensitive the SFR-TSI relationship is to the consideration. We allowed a 0.2 dex enhancement in SF in the range of $10 \%-30 \%$ of galaxies to mimic it and found that our results are reasonably stable against the possibility.

\subsection{Predictions at Higher Redshifts}

In our analysis based on the SFR-TSI relation, lower values of SFR are results of a longer time spent inside clusters and thus provide information on the quenching process at higher redshifts. A problem here is that lower values of SFR are associated with larger uncertainties in the measurement, making the exploration of high-redshift processes more difficult. In principle, our analysis makes use of the measurement errors of the observed data, and so the larger uncertainties at higher redshifts are implicitly being considered. However, the uncertainties at high redshifts are not only on the observed data but also on the theoretical assumptions made in the analysis. For example, the redshift dependence of stellar feedback, AGN feedback, and merger frequency and so on may all be influencing each other in nonlinear ways. These are admittedly too difficult to disentangle through our simplistic formalism. Having said that, one very certain way of improving the situation is to achieve smaller uncertainties in the SFR of galaxies. Lowering the uncertainties by a factor of a few may help us constrain the timescales and associated parameters (e.g., $\alpha$ ) much better. Alternatively, if we can measure the SFRs of the galaxies of clusters at higher redshifts (e.g., $z=0.5$ ), we would be able to explore the quenching history of galaxies farther back in time, eventually completing the story of their quenching.

\section{Summary and Conclusion}

We attempted to clarify the quenching mechanisms for disk galaxies in cluster environments using simulated (YZiCS) and observed (SDSS; Tempel et al. 2014) cluster samples in this study. The main method of performing the analysis is to derive the SFR-TSI relationship for disk cluster galaxies at $z=0.08$ using both cluster samples (Section 4.1) and to look for the best quenching model that matches the empirical SFR-TSI relationship (Section 4.2). To derive such relationships, we used the distribution of galaxies in the phase-space diagram in which galaxies have a good geographic trend with their physical properties (TSI and SFR in this analysis; Figure 4). Moreover, we divided the sample by stellar mass and by the host mass in order to examine the dependence on those variables. Our methodology can be summarized as follows.

1. Before deriving the distribution of galaxies in phase space, we carefully defined the normalization coefficients and coordinates of the projected phase-space diagram for both simulated and observed satellite galaxies (Sections 2.2.2 and 3.1). Furthermore, we imposed a boundary on the projected phase-space diagrams that ensures there is little contamination from foreground/background objects (Figure 2).

2. We then constructed a density function of the TSI and SFR of disk satellite galaxies within different pixels in the projected phase space (Figure 4 and Sections 3.2 and 3.3). TSI density functions are generated using the YZiCS cluster samples, while SFR ones are derived from the SDSS cluster samples (Tempel et al. 2014) combined with the physical properties of galaxies (Salim et al. 2016).

3. With the key assumptions (Section 4.1), we associated the TSI and SFR density functions within a pixel using an abundance-matching approach (Figure 4), thus deriving the SFR-TSI relationship at $z=0.08$ (Figure 8).

4. We parameterized the SFH for infalling galaxies based on a quenching model (Equation (6)). The quenching model basically comprises three main phases: (i) the ex situ quenching phase, in which galaxies are in the pre-infall stage and their SFRs decrease with ex situ quenching timescale $\left(\tau_{\text {ex situ }}\right)$; (ii) the delay phase, in which galaxies are accreted to clusters, but their SFR continues to evolve in the same manner as the ex situ quenching phase for the delay time $\left(t_{\mathrm{d}}\right)$; and (iii) the cluster-quenching phase, in which the SFRs of galaxies show a sudden drop after the 
delay phase on the cluster-quenching timescale $\left(\tau_{\text {cluster }}\right)$. (See Figure 6 for an illustration of the model).

5. Based on the quenching model, we split the sample by stellar mass and try to empirically constrain the quenching parameters of the model by attempting to recover the SFR-TSI relationship as closely as possible (Section 4.2).

Then, our main conclusions are as follows.

1. We do not see a clear trend with cluster mass in the SFRTSI relationships (Figure 7). We cannot test the presence of cluster mass dependence effectively because YZiCS does not provide a sufficiently large number of clusters for which TSI is measured.

2. The ex situ quenching timescale ( $\tau_{\text {ex situ }}$; Section 5.1$)$ is constrained to be 2.5-3.5 Gyr, decreasing with increasing stellar mass, $\tau_{\text {ex situ }} \propto M_{*}^{-0.45}$. This value is fairly comparable with that of previous studies (e.g., Noeske et al. 2007). The stellar mass trend indicates that massive galaxies can be significantly self-quenched prior to their infall into clusters. Cumulative mass quenching for those galaxies or gas depletion with a short timescale in more massive galaxies (e.g., Boselli et al. 2014a; Saintonge et al. 2017) might be the main contributor (e.g., Roberts et al. 2019). Moreover, the values of the ex situ quenching timescale are generally lower than those of field samples, likely indicating the role of preprocessing at play in the galaxy group environments.

3. Delay times $\left(t_{\mathrm{d}}\right.$; Section 5.2) are measured around $2 \mathrm{Gyr}$ regardless of stellar mass (Figure 10, panel (d)), similar to the typical crossing time of clusters (Section 5.2; e.g., Mok et al. 2014; Tal et al. 2014; Fossati et al. 2017; Lemaux et al. 2019). This indicates that, during the first pericenter passage, quenching inside clusters might not be strong enough for galaxies to show up in different decreasing rates of SF. During this time, only a gentle mode of quenching, such as neutral and hot gas stripping, may occur.

4. The cluster-quenching timescales ( $\tau_{\text {cluster }}$; Section 5.3) are measured to be $0.7-1.5 \mathrm{Gyr}$, decreasing with increasing stellar mass. The small values of the clusterquenching timescales imply a quick and strong quenching process in action. We suspect that it is ram pressure stripping which acts on both neutral and molecular gases. We explain the inverse mass dependence of clusterquenching timescale by arguing that it may be due to the fact that the ISM distribution is more centrally concentrated in lower mass galaxies and hence more resistant against ram pressure.

The purpose of this investigation is to understand the main processes that caused cluster galaxies to be SF quenched. Our new approach of combining the empirical SFRs and theoretical TSI through abundance matching has allowed us to measure the quenching timescales outside and inside clusters, and the delay time that bridges the two. The quenching timescales inside clusters are the shortest of the three. If the quenching rate (i.e., timescale) is considered important, this means that the processes acting inside clusters are the most important processes that caused cluster galaxies to be passive.

The quenching timescales outside clusters are longer and so seemingly less important; however, there are two aspects one should not miss. First, the cumulative amount of SF quenching can easily be much larger in the case of ex situ quenching, simply because the time galaxies spend outside clusters can be much larger than the cluster-quenching timescales. Besides, it sets an important mass trend on the gas fraction of galaxies when they enter clusters. Galaxies with a lower gas fraction tend to lose their gas more easily. Consequently, the mass trend of ex situ quenching provides a seed for the inverse mass dependence of the cluster-quenching timescale. In other words, the nature of the quenching process (amount and mass dependence) outside clusters in a way provides a guideline for how quickly cluster quenching must take place later.

Our analysis is limited to reconstructing the SFH of cluster galaxies only for the last few gigayears (depending on galaxy mass), because, in our simple paradigm, galaxies entering clusters earlier than that would have SFRs that are too low to be measured empirically using the SED-fitting approach employed in this study. We can explore earlier times by achieving measurements of lower SFRs than currently available through all-sky surveys (e.g., SDSS) or more directly by measuring SFRs of galaxies in clusters at higher redshifts (e.g., $z=0.5$ ). This will be an interesting observational challenge in the future. From the theoretical side, we have not separated preprocessing effects for group infallers from cluster processes. As we have discovered through this investigation, what happens before cluster entry has a profound impact on what happens after. It will be a natural next step to try to assess the significance of preprocessing in making passive cluster galaxies.

We thank Aeree Chung, Ivy Wong, and Taysun Kimm for the constructive feedback. S.K.Y. acted as the corresponding author and acknowledges support from the Korean National Research Foundation (NRF-2017R1A2A05001116). The supercomputing time for the numerical simulation was kindly provided by KISTI to S.K.Y. (KSC-2014-G2-003), and large data transfer was supported by KREONET, which is managed and operated by KISTI. H.C. acknowledges the support by Norwegian Research Council Young Research Talents Grant 276043, "Simulating the Circumgalactic Medium and the Cycle of Baryons In and Out of Galaxies Throughout Cosmic History."

\section{ORCID iDs}

Jinsu Rhee (1) https://orcid.org/0000-0002-0184-9589

Hoseung Choi (i) https://orcid.org/0000-0001-7229-0033

Sukyoung K. Yi (iD https://orcid.org/0000-0002-4556-2619

\section{References}

Adhikari, S., Dalal, N., More, S., \& Wetzel, A. 2019, ApJ, 878, 9 Ahn, C. P., Alexandroff, R., Allende, P. C., et al. 2014, ApJS, 211, 17 Aubert, D., Pichon, C., \& Colombi, S. 2004, MNRAS, 352, 376 Balogh, M. L., Eke, V., Miller, C., et al. 2004, MNRAS, 348, 1355 Balogh, M. L., McGee, S. L., Mok, A., et al. 2016, MNRAS, 456, 4364 Balogh, M. L., Navarro, J. F., \& Morris, S. L. 2000, ApJ, 540, 113 Behroozi, P. S., Wechsler, R. H., Hearin, A. P., \& Conroy, C. 2019, MNRAS, 488,3143

Behroozi, P. S., Wechsler, R. H., Lu, Y., et al. 2014, ApJ, 787, 156

Bekki, K. 2014, MNRAS, 438, 444

Binney, J. 1977, ApJ, 215, 483

Birnboim, Y., \& Dekel, A. 2003, MNRAS, 345, 349

Boselli, A., Cortese, L., Boquien, M., et al. 2014a, A\&A, 564, 66

Boselli, A., Cortese, L., Boquien, M., et al. 2014b, A\&A, 564, 67

Boselli, A., Rohelly, Y., Fossati, M., et al. 2016, A\&A, 596, 11

Casoli, F., Sauty, S., Gerin, M., et al. 1998, A\&A, 331, 451

Chabrier, G. 2003, PASP, 115, 763

Choi, H., \& Yi, S. 2017, ApJ, 837, 68 
Chung, A., van Gorkom, J. H., Kenney, J. D. P., \& Vollmer, B. 2007, ApJ, 659,115

Contini, E., Kang, X., Romeo, A. D., et al. 2017, ApJ, 849, 156 Cortese, L., Boissier, S., Boselli, A., et al. 2012, A\&A, 544, 101 Croton, D. J., Springel, V., White, S. D., et al. 2006, MNRAS, 365, 11 Darvish, B., Mobasher, B., Sobral, D., et al. 2016, ApJ, 825, 113 De Lucia, G., Weinmann, S., Poggianti, B. M., et al. 2012, MNRAS, 423, 1277 Dressler, A. 1980, ApJ, 236, 351

Dubois, Y., Devriendt, J., Slyz, A., \& Teyssier, R. 2012, MNRAS, 420, 2662 Dubois, Y., Peirani, S., Pichon, C., et al. 2016, MNRAS, 463, 3948 Dubois, Y., Pichon, C., Welker, C., et al. 2014, MNRAS, 444, 1453 Einasto, M., Deshev, B., Lietzen, H., et al. 2018a, A\&A, 610, 82 Einasto, M., Gramann, M., Park, C., et al. 2018b, A\&A, 620, 149 Foltz, R., Wilson, G., Muzzin, A., et al. 2018, A\&A, 866, 136 Fossati, M., Mendel, J. T., Bosseli, A., et al. 2018, A\&A, 614, 57 Fossati, M., Wilman, D. J., Mendel, J. T., et al. 2017, ApJ, 835, 153 Fumagalli, M., Krumholz, M. R., Prochaska, J. X., et al. 2009, ApJ, 697, 1811 Gao, L., White, S. D. M., Jenkins, A., et al. 2004, MNRAS, 355, 819 Gavazzi, G. 1987, ApJ, 320, 96

Gerke, B. F., Newman, J. A., Faber, S. M., et al. 2007, MNRAS, 376, 1425

Gill, S. P. D., Knebe, A., \& Gibson, B. K. 2005, MNRAS, 356, 1327 Girardi, M., Giuricin, G., Mardirossian, F., et al. 1998, ApJ, 505, 74 Gómez, P. L., Nichol, R. C., Miller, C. J., et al. 2003, ApJ, 584, 210 Gunn, J. E., \& Gott, J. R., III 1972, ApJ, 176, 1

Haines, C. P., Pereira, M. J., Smith, G. P., et al. 2013, ApJ, 775, 126

Haines, C. P., Pereira, M. J., Smith, G. P., et al. 2015, ApJ, 806, 101

Han, S., Smith, R., Choi, H., et al. 2018, ApJ, 866, 78

Hearin, A. P., \& Watson, D. F. 2013, MNRAS, 435, 1313

Hernández-Fernández, J. D., Haines, C. P., Diaferio, A., et al. 2014, MNRAS, 438, 2186

Hogg, D. W., Blanton, M. R., Brinchmann, J., et al. 2004, ApJ, 601, 29

Huertas-Company, M., Aguerri, J. A. L., Bernardi, M., et al. 2011, A\&A, 525,157

Jaffé, Y. L., Poggianti, B. M., Moretti, A., et al. 2018, MNRAS, 476, 4735

Jaffé, Y. L., Smith, R., Candlish, G. N., et al. 2015, MNRAS, 448, 1715

Jung, S. L., Choi, H., Wong, O. I., et al. 2018, ApJ, 865, 156

Kauffmann, G., White, S. D. M., Heckman, T. M., et al. 2004, MNRAS, 353,713

Kaviraj, S., Laigle, C., Kimm, T., et al. 2017, MNRAS, 467, 4739

Kelkar, K., Gray, M. E., Aragón-Salamanca, A., et al. 2019, MNRAS, 486, 868

Kereš, D., Katz, N., Weinberg, D. H., \& Davé, R. 2005, MNRAS, 363, 2

Kimm, T., Somerville, R. S., Yi, S. K., et al. 2009, MNRAS, 394, 1131

Komatsu, E., Smith, K. M., Dunkley, J., et al. 2011, ApJS, 192, 18

Koopmann, R. A., \& Kenney, J. D. P. 2004, ApJ, 613, 866

Larson, R. B. 1974, MNRAS, 169, 229

Larson, R. B., Tinsley, B. M., \& Caldwell, C. N. 1980, ApJ, 237, 692

Lee, B., \& Chung, A. 2018, ApJ, 866, 10

Lee, B., Chung, A., Tonnesen, S., et al. 2017, MNRAS, 466, 1382

Lee, J., Kim, S., Jeong, H., et al. 2018, ApJ, 864, 69

Lemaux, B. C., Tomczak, A. R., Lubin, L. M., et al. 2019, MNRAS, 490, 1231

Lewis, I., Balogh, M. L., De Propris, R., et al. 2002, MNRAS, 334, 673

Lidman, C., Rosati, P., Tanaka, M., et al. 2008, A\&A, 489, 981

Limousin, M., Sommer-Larsen, J., Natarajan, P., \& Milvang-Jensen, B. 2009, ApJ, 696, 1771

Lisker, T., Vijayaraghavan, R., Janz, J., et al. 2018, ApJ, 865, 40

Lotz, M., Remus, R., Dolag, K., et al. 2019, MNRAS, 488, 5370

Madau, P., \& Dickinson, M. 2014, ARA\&A, 52, 415

Mahajan, S., Mamon, G. A., \& Raychaudhury, S. 2011, MNRAS, 416, 2882

Maier, C., Ziegler, B. L., Haines, C. P., \& Smith, G. P. 2019, A\&A, 621, 131

McCarthy, I. G., Frenk, C. S., Font, A. S., et al. 2008, MNRAS, 383, 593

McGee, S. L., Balogh, M. L., Wilman, D. J., et al. 2011, MNRAS, 413, 996

McGee, S. L., Bower, R. G., \& Balogh, M. 2014, MNRAS, 442, 105

Mihos, J. C. 2004, in Cluster of Galaxies: Probes of Cosmological Structure and Galaxy Evolution, ed. J. S Mulchaey, A. Dressler, \& A. Oemler (Cambridge: Cambridge Univ. Press), 277
Mistani, P. A., Sales, L. V., Pillepich, A., et al. 2016, MNRAS, 455, 2323

Mok, A., Balogh, M. L., McGee, S. L., et al. 2013, MNRAS, 431, 1090

Mok, A., Balogh, M. L., McGee, S. L., et al. 2014, MNRAS, 438, 3070

Moore, B., Katz, N., Lake, G., et al. 1996, Natur, 379, 613

Moore, B., Lake, G., \& Katz, N. 1998, ApJ, 495, 139

Muzzin, A., Labbé, I., Franx, M., et al. 2012, ApJ, 761, 142

Muzzin, A., van der Burg, R. F. J., McGee, S. L., et al. 2014, ApJ, 796, 65

Nantais, J. B., Muzzin, A., van der Burg, R. F. J., et al. 2017, MNRAS, 465,104

Navarro, J. F., Frenk, C. S., \& White, S. D. M. 1996, ApJ, 462, 563

Noble, A. G., Webb, T. M. A., Muzzin, A., et al. 2013, ApJ, 768, 118

Noble, A. G., Webb, T. M. A., Yee, H. K. C., et al. 2016, ApJ, 816, 48

Noeske, K. G., Faber, S. M., Weiner, B. J., et al. 2007, ApJ, 660, 47

Oman, K. A., \& Hudson, M. J. 2016, MNRAS, 463, 3083

Oman, K. A., Hudson, M. J., \& Behroozi, P. S. 2013, MNRAS, 431, 2307

Paccagnella, A., Vulcani, B., Poggianti, B. M., et al. 2016, ApJ, 816, 25

Pallero, D., Gómez, F. A., Padilla, N. D., et al. 2019, MNRAS, 488, 847

Pasquali, A., Smith, R., Gallazzi, A., et al. 2019, MNRAS, 484, 1702

Peng, Y., Lilly, S. J., Kovač, K., et al. 2010, ApJ, 721, 193

Postman, M., Franx, M., Cross, N. J. G., et al. 2005, ApJ, 623, 721

Rhee, J., Smith, R., Choi, H., et al. 2017, ApJ, 843, 128

Roberts, I. D., Parker, L. C., Brown, T., et al. 2019, ApJ, 873, 42

Rodriguez-Gomez, V., Sales, L. V., Genel, S., et al. 2017, MNRAS, 467, 3083

Rodríguez-Muñoz, L., Rodighiero, G., Mancini, C., et al. 2019, MNRAS, 485,586

Saintonge, A., Catinella, B., Tacconi, L. J., et al. 2017, ApJS, 233, 22

Salim, S., Lee, J. C., Janowiecki, S., et al. 2016, ApJS, 227, 2

Sheen, Y.-K., Yi, S. K., Ree, C. H., \& Lee, J. 2012, ApJS, 202, 8

Smith, R., Choi, H., Lee, J., et al. 2016, ApJ, 833, 109

Smith, R., Davies, J. I., \& Nelson, A. H. 2010, MNRAS, 405, 1723

Smith, R., Pacifici, C., Pasquali, A., \& Calderon-Castillo, P. 2019, ApJ, 876,145

Smith, R., Sánchez-Janssen, R., Beasley, M. A., et al. 2015, MNRAS, 454, 2502

Smith, R., Sánchez-Janssen, R., Fellhauer, M., et al. 2013, MNRAS, 429, 1066

Snyder, G. F., Torrey, P., Lotz, J. M., et al. 2015, MNRAS, 454, 1886

Steinhauser, D., Schindler, S., \& Springel, V. 2016, A\&A, 591, 51

Tacchella, S., Dekel, A., Carollo, C. M., et al. 2016, MNRAS, 458, 242

Tal, T., Dekel, A., Oesch, P., et al. 2014, ApJ, 789, 164

Taranu, D. S., Hudson, M. J., Balogh, M. L., et al. 2014, MNRAS, 440, 1943

Tempel, E., Tamm, A., Gramann, M., et al. 2014, A\&A, 566, 1

Teyssier, R. 2002, A\&A, 385, 337

Tomczak, A. R., Lemaux, B. C., Lubin, L. M., et al. 2018, MNRAS, 484, 4695

Tomczak, A. R., Quadri, R. F., Tran, K. H., et al. 2016, ApJ, 817, 118

Toomre, A., \& Toomre, J. 1972, ApJ, 178, 623

Tweed, D., Devriendt, J., Blaizot, J., et al. 2009, A\&A, 506, 647

Tyler, K. D., Rieke, G. H., \& Bai, L. 2013, ApJ, 773, 86

von der Linden, A., Wild, V., Kauffmann, G., et al. 2010, MNRAS, 404, 1231

Vulcani, B., Poggianti, B. M., Finn, R. A., et al. 2010, ApJ, 710, 1

Vulcani, B., Poggianti, B. M., Gullieuszik, M., et al. 2018, ApJ, 866, 25

Wang, L., Li, C., Kauffmann, G., \& De Lucia, G. 2007, MNRAS, 377, 1419

Weinmann, S. M., Kauffmann, G., van den Bosch, F. C., et al. 2009, MNRAS, 394, 1213

Weinmann, S. M., Kauffmann, G., von der Linden, A., \& De Lucia, G. 2010, MNRAS, 406, 2249

Wetzel, A. R. 2011, MNRAS, 412, 49

Wetzel, A. R., Tinker, J. L., Conroy, C., \& van den Bosch, F. C. 2013, MNRAS, 432, 336

Wheeler, C., Phillips, J. I., Cooper, M. C., et al. 2014, MNRAS, 442, 1396

Whitaker, K. E., van Dokkum, P. G., Brammer, G., \& Franx, M. 2012, ApJ, 754, 29

Wolf, C., Aragón-Salamanca, A., Balogh, M., et al. 2009, MNRAS, 393, 1302

Woo, J., Dekel, A., Faber, S. M., et al. 2013, MNRAS, 428, 3306

Yi, S. K., Lee, J., Jung, I., et al. 2013, A\&A, 554, 122

Yoon, H., Chung, A., Smith, R., \& Jaffé, Y. L. 2017, ApJ, 838, 81 